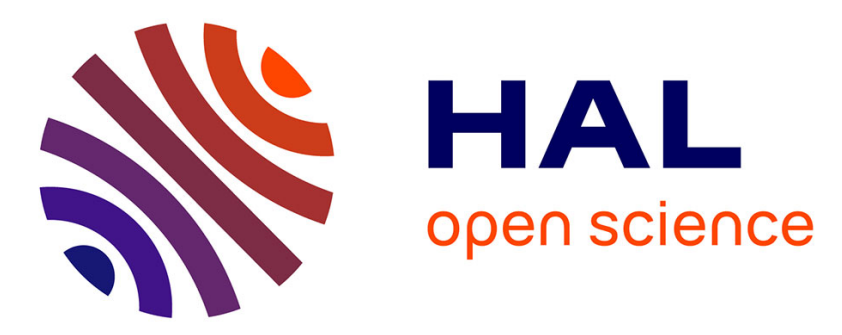

\title{
Adsorption of small inorganic molecules on a defective MoS_2 monolayer
}

Cesar Gonzalez, Blanca Biel, Yannick J Dappe

\section{To cite this version:}

Cesar Gonzalez, Blanca Biel, Yannick J Dappe. Adsorption of small inorganic molecules on a defective MoS_2 monolayer. Physical Chemistry Chemical Physics, 2017, 19, pp.9485-9499. 10.1039/C7CP00544J . cea-01533715

\section{HAL Id: cea-01533715 https://hal-cea.archives-ouvertes.fr/cea-01533715}

Submitted on 6 Jun 2017

HAL is a multi-disciplinary open access archive for the deposit and dissemination of scientific research documents, whether they are published or not. The documents may come from teaching and research institutions in France or abroad, or from public or private research centers.
L'archive ouverte pluridisciplinaire HAL, est destinée au dépôt et à la diffusion de documents scientifiques de niveau recherche, publiés ou non, émanant des établissements d'enseignement et de recherche français ou étrangers, des laboratoires publics ou privés. 
Check for updates

Cite this: Phys. Chem. Chem. Phys., 2017, 19, 9485

Received 24th January 2017. Accepted 14th March 2017

DOI: $10.1039 / c 7 c p 00544 j$

rsc.li/pccp

\title{
Adsorption of small inorganic molecules on a defective $\mathrm{MoS}_{2}$ monolayer $\dagger$
}

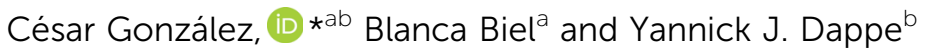

\begin{abstract}
We present a theoretical study of molecular adsorption on defects on a $\mathrm{MoS}_{2}$ monolayer. Based on Density Functional Theory, our calculations confirm that small inorganic molecules, such as $\mathrm{CO}_{2}, \mathrm{CO}, \mathrm{H}_{2} \mathrm{O}, \mathrm{NO}$, $\mathrm{NO}_{2}, \mathrm{H}_{2}$ and $\mathrm{N}_{2}$, remain bonded to the pristine monolayer through weak van der Waals interactions, suggesting that the molecules may easily diffuse over the clean monolayer. On the other hand, the introduction of defects can lead to three different situations, depending on the defect and the molecule considered: physisorption, chemical (strong) bonding to the metallic defects, namely the Mo substitutional atoms on the $\mathrm{S}$ vacancies, and dissociation, that can take place spontaneously at $0 \mathrm{~K}$ in some specific cases or by the effect of thermal agitation in molecules such as $\mathrm{CO}_{2}$ or $\mathrm{NO}_{2}$ on the $\mathrm{S}$ vacancy. Our energetic and electronic analyses provide an explanation to such bonding possibilities, showing that in the low interacting situations, the molecules tend to adopt a planar configuration parallel to the monolayer, while a molecular rotation is favored in order to facilitate the bond formation on the reactive sites. Finally, the ab initio based Scanning Tunneling Microscopy (STM) simulations show the fingerprint of each molecule adsorbed on the most reactive site. This work opens the way to the possibility of tuning the catalytic properties of $\mathrm{MoS}_{2}$ by controlling the creation of specific defects in the $\mathrm{MoS}_{2}$ monolayer.
\end{abstract}

\section{Introduction}

The isolation of graphene ${ }^{1}$ has opened a fantastic field of new research in the bidimensional world. Indeed, beyond the fantastic properties of the honeycomb carbon monolayer, researchers have seen a rising interest in a new family of two dimensional (2D) materials. Together with graphene, boron-nitride (h-BN), ${ }^{2}$ fluorographene, ${ }^{3}$ or the general transition metal dichalcogenide structures (TMDC) ${ }^{4-7}$ have become the new playground in Physics, Chemistry, Biology or Materials Science.

For sure the new electronic properties in the new born 2D world, like the Klein tunneling in graphene ${ }^{8}$ or the strainmediated direct to indirect bandgap transition in the TMDC $\mathrm{MoSe}_{2}{ }^{7}$ have attracted a lot of attention. However, the properties related to electronic transport or optoelectronics are not the

\footnotetext{
${ }^{a}$ Departamento de Electrónica y Tecnología de Computadores, Campus de Fuente Nueva \& CITIC, Universidad de Granada, Campus de Aynadamar, E-18071 Granada, Spain. E-mail: cesar.gonzalez.pascual@gmail.com

${ }^{b}$ SPEC, CEA, CNRS, Université Paris-Saclay, CEA Saclay 91191 Gif-sur-Yvette Cedex, France

$\dagger$ Electronic supplementary information (ESI) available: Movies showing the atomic movement for different structures calculated at room temperature during 30 ps: (1) the defective $\mathrm{MoS}_{2}$ structure formed by two Mo atoms occupying the $\mathrm{S}$ divacancy. (2) The dissociation of a $\mathrm{CO}_{2}$ molecule on the defect formed by two Mo atoms occupying the $\mathrm{S}$ divacancy. (3) The dissociation of a $\mathrm{CO}_{2}$ molecule on a $\mathrm{S}$ vacancy. (4) The dissociation of a $\mathrm{NO}_{2}$ molecule on a $\mathrm{S}$ vacancy. See DOI: $10.1039 / \mathrm{c} 7 \mathrm{cp} 00544 \mathrm{j}$
}

only areas with potential innovative applications. For example, another important field of research, merging the world of $2 \mathrm{D}$ materials with that of surface physics, is the study of molecular adsorption properties on 2D materials. Hence, the interaction between 2D materials and molecules can be exploited for molecular storage, within the frame of energy recovery with dihydrogen for example. ${ }^{9}$ Those systems can also be developed for more environmental friendly devices, like catalytic converters for car manufacture or for chemistry applications like reaction catalyzers. For such purposes, the interaction between a molecule and the selected 2D material should be thoroughly described.

It is well known now that $2 \mathrm{D}$ materials generally tend to be poorly reactive even though the monolayer properties can be altered and the shape of the flake can be controlled by, for instance, an oxidation process at the edges. ${ }^{10}$ Based on this idea, the molecular adsorption on 2D materials finds applications in electronic devices like gas sensors. ${ }^{11,12}$ Many authors have fabricated $\mathrm{MoS}_{2}$ transistors in order to validate their potential use in such kind of devices. ${ }^{13-15}$ Indeed, the presence of physisorbed molecules on the $\mathrm{MoS}_{2}$ monolayer produces a change in the electronic properties and consequently a change in the electronic current in the transistor, leading to the molecular detection. The low interaction was proved theoretically by Zhao et al. for a wide range of small molecules on the perfect $\mathrm{MoS}_{2}$ monolayer. ${ }^{16}$ The calculations confirmed that the molecules were physisorbed on the surface at a typical van der Waals (vdW) distance in most cases, even though some molecules 
experienced a stronger interaction with the monolayer, ${ }^{17}$ in good agreement with experimental evidence obtained in the gas sensing studies. ${ }^{13-15}$ This low interaction has been very recently confirmed in a new study involving $\mathrm{CO}_{2}$ molecules deposited on a $\mathrm{MoS}_{2}$ monolayer. ${ }^{18}$

On the other hand, reactivity and transport properties of $\mathrm{MoS}_{2}$ can be influenced by defects like vacancies or substitutional atoms. ${ }^{19,20}$ In both works, the authors studied the different kinds of defects present on a $\mathrm{MoS}_{2}$ monolayer by means of Scanning Transmission Electron Microscopy (STEM) experiments and Density Functional Theory (DFT) simulations. The atomic resolution obtained in the STEM measurements allowed them to define the different defects in the samples, while a detailed analysis of the formation energy of each case was performed by means of DFT calculations. Hong et al. showed that the formation of the defects strongly depends on the experimental technique used for the monolayer growth, obtaining a larger number of $S$ vacancies in the samples grown by the Mechanical Exfoliation (ME) and Chemical Vapor Deposition (CVD) techniques, while the Mo substitutional cases were found more frequently in the samples grown by the Physical Vapor Deposition (PVD) technique. These defects could interact strongly with the molecules, leading to an important change in the electronic transport. This effect is of crucial relevance and should be analyzed. Following this idea, Li et al. proposed an increase of the number of defects in 2D materials, in order to enhance their reactivity, ${ }^{21}$ while Makarova et al. have recently studied by means of DFT calculations the healing of S-defects through thiol molecule adsorption and the subsequent breakup of the S-C bond. ${ }^{22}$

This stronger interaction with the defective structures can lead to molecular chemisorption or dissociation as it was previously demonstrated in other materials such as $\mathrm{H}_{2} \mathrm{O}$ molecules on $\mathrm{TiO}_{2}$ defects ${ }^{23}$ or $\mathrm{NO}_{2}$ molecules on $\mathrm{ZnO}$ defects. ${ }^{24}$ These potential dissociative processes have been theoretically studied for $\mathrm{CO}_{2}, \mathrm{H}_{2}, \mathrm{O}_{2}$ and $\mathrm{CO}$ molecules on graphene/silicene defects. ${ }^{25,26}$ Very recently, different groups have analyzed the interactions of some molecules in a defective $\mathrm{MoS}_{2}$ monolayer. ${ }^{27-32}$ The first two works show both experimentally and theoretically the molecular chemisorption or dissociation for $\mathrm{O}_{2}$ molecules on $\mathrm{MoS}_{2}$ defects. Li et al. extended the study to other molecules on a $\mathrm{S}$ vacancy, ${ }^{29}$ while Sahoo and coworkers applied a similar analysis for an antisite, i.e. a Mo atom occupying a $\mathrm{S}$ vacancy. ${ }^{30} \mathrm{~A}$ deep analysis of the interaction of the $\mathrm{CO}_{2}$ and $\mathrm{CH}_{3}$ molecules with different $\mathrm{MoS}_{2}$ defects has been performed by Yu et al., ${ }^{31}$ and finally, a great reactivity enhancement has been tested in the $\mathrm{MoS}_{2}$ edges using $\mathrm{NO}_{2}$ molecules. ${ }^{32}$

Following our previous works on defective $\mathrm{MoS}_{2},{ }^{33,34}$ we present here a theoretical study of molecular adsorption on a $\mathrm{MoS}_{2}$ monolayer in the presence of point defects. We show that it is possible to use them in order to produce molecular chemisorption at the TMDC layer. We analyze the different molecule-defect combinations that can lead to either the formation of a weak vdW interaction, allowing the diffusion over the $\mathrm{MoS}_{2}$, or a stronger covalent bond that could fix the molecule on the defect. Finally we study the potential molecular dissociation on the different $\mathrm{MoS}_{2}$ sites. Our results suggest the possibility of creating specific defects to favor on-surface molecular chemistry.

\section{Theoretical details}

DFT simulations have been performed using the plane wave Vienna $a b$ initio Simulation Package (VASP) code. ${ }^{35}$ VASP uses the pseudo-potential approximation following the Projector Augmented Wave (PAW) approach. ${ }^{36}$ In our simulations, the PBE functional for the Gradient Generalized Approximation of the exchange and correlation potential ${ }^{37}$ has been chosen and the energy cutoff of the plane waves is fixed at $400 \mathrm{eV}$, the highest default value of the different elements as given by the pseudo-potentials provided by VASP. In all cases considered here, the vacuum layer set for the supercell calculations is fixed at $15 \AA$, which has been proved to be enough to avoid undesired interactions between neighbouring supercells in the perpendicular direction. The valence electrons for the different elements used in the calculations are: 6 for S ( $43 \mathrm{p}$ and $23 \mathrm{~s}$ ), 14 for Mo ( 4 4d, $25 \mathrm{~s}, 64 \mathrm{p}$ and $24 \mathrm{~s}), 4$ for C (2 2p and $22 \mathrm{~s}$ ), 6 for O (4 2p and $22 \mathrm{~s}$ ), 5 for $\mathrm{N}$ ( $32 \mathrm{p}$ and $22 \mathrm{~s}$ ) and 1 for $\mathrm{H}(1 \mathrm{~s})$.

Within that frame, we have obtained a lattice parameter of $3.185 \AA$ for a $1 \times 1$ unit cell and a S-S vertical distance of $3.12 \AA$ between both $\mathrm{S}$ sub-layers, in good agreement with experimental evidence ${ }^{38}$ and previous simulations. ${ }^{33,39,40}$ Then, a rectangular unit cell of $\mathrm{MoS}_{2}$ formed by 72 atoms (48 S and 24 Mo atoms) has been considered (see the ball and stick model in Fig. 1(a)). This unit cell can be repeated in both $X$ and $Y$ directions, leading to the periodic boundary conditions required by the DFT methodology. Thus, the different $\mathrm{MoS}_{2}$ defects (single Mo and S vacancy, S di-vacancy and the corresponding substitutional cases, i.e. one or two Mo atoms in the $\mathrm{S}$ divacancy, one or two $\mathrm{S}$ atoms in the Mo vacancy and one Mo atom in the $\mathrm{S}$ mono-vacancy) have been created in this rectangular unit cell. All the atomic configurations were previously optimized and the characterization of their electronic and geometrical properties by means of Scanning Tunneling Microscopy (STM) and Atomic Force Microscopy (AFM) simulations can be found elsewhere. ${ }^{33,34}$ For this reason, we will mainly focus our attention on the energetic stability and only the most important structural features will be mentioned here. For a monolayer formed by $N_{\text {Mo }}$ and $N_{\mathrm{S}}$ atoms, the formation energy can be expressed as ${ }^{41}$

$$
E_{\mathrm{f}}=E_{\mathrm{t}}-N_{\mathrm{Mo}} E(\mathrm{Mo})-N_{\mathrm{S}} E(\mathrm{~S})
$$

where $E(\mathrm{Mo})$ and $E(\mathrm{~S})$ are the atomic energy of a single Mo or S atom, respectively, in the pristine $\mathrm{MoS}_{2}$ monolayer. The final total energies $\left(E_{\mathrm{t}}\right)$ obtained in our simulations can be used to calculate the formation energy for each defective structure and consequently $N_{\text {Mo }}$ and $N_{\mathrm{S}}$ correspond to the number of Mo and $S$ atoms in our rectangular unit cell plus the extra atoms in the substitutional sites $\left(\mathrm{Sub}_{\mathrm{Mo} / \mathrm{s}}\right)$ and minus the atoms removed from the vacancies $\left(\mathrm{V}_{\mathrm{Mo} / \mathrm{s}}\right)$. Thus, eqn (1) can be rewritten as

$$
E_{\mathrm{f}}=E_{\mathrm{t}}-E_{\text {pristine }}+\mathrm{V}_{\mathrm{Mo} / \mathrm{S}} \mu(\mathrm{Mo} / \mathrm{S})-\mathrm{Sub}_{\mathrm{Mo} / \mathrm{s}} \mu(\mathrm{Mo} / \mathrm{S})
$$



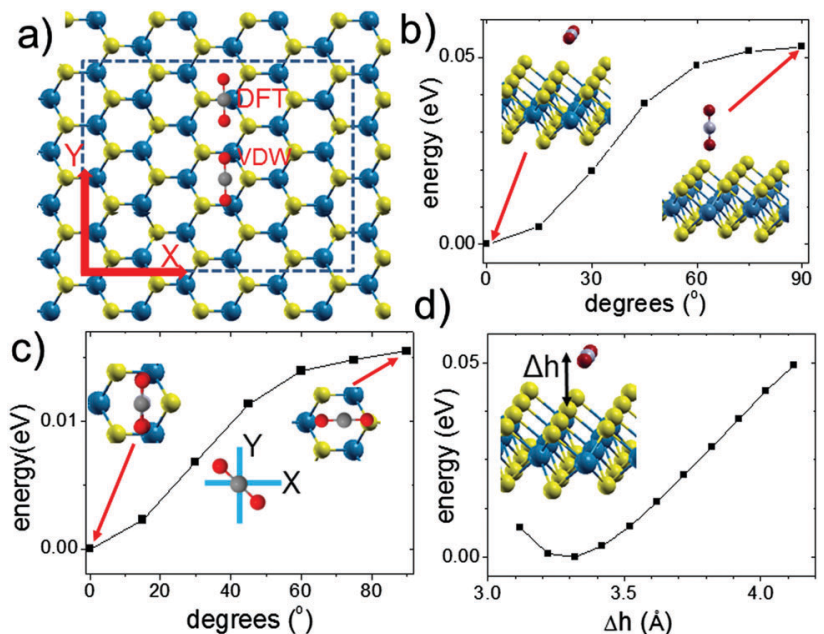

Fig. 1 (a) Frontal view of the ball and stick model of the rectangular $\mathrm{MoS}_{2}$ unit cell used in the calculations. $\mathrm{A} \mathrm{CO}_{2}$ molecule is adsorbed on the most favorable sites with and without the inclusion of vdW interactions (labelled with VDW and DFT respectively). The dotted line shows the borders of the unit cell while the blue arrows indicate the $X$ and $Y$ directions. The yellow/blue spheres correspond to $\mathrm{S} / \mathrm{Mo}$ atoms and red/grey to O/C. Energy of the most stable case as a function of (b) the molecular rotation in the $Y Z$ plane (in the inset the lateral view of the equilibrium geometry of a $\mathrm{CO}_{2}$ molecule adsorbed for a configuration parallel to the $\mathrm{S}$ row and a configuration perpendicular to the plane of the monolayer, respectively), (c) the molecular rotation in the $X Y$ plane with respect to the most stable VDW configuration (in the inset two molecular rotations in the same plane) and (d) the molecule- $\mathrm{MoS}_{2}$ distance defined by the black arrow. Energies and 0 degree geometry are referred to the orientation labeled with VDW.

where $E_{\text {pristine }}$ is the energy of the perfect unit cell of Fig. 1(a) and $\mu(\mathrm{Mo} / \mathrm{S})$ is the chemical potential of Mo or S. A specific discussion on the atomic energy is provided in the next section.

Once the most stable structures have been determined, several small inorganic molecules are adsorbed in different orientations over the most relevant points, both onto the pristine monolayer (on top of a Mo or S atom, on the bridge and hollow sites and on the center of the hexagon) and over all the defects mentioned before. Thus, the system is fully relaxed using $16 k$-points calculated within the centered Monkhorst-Pack scheme in the first Brillouin zone ${ }^{42}$ and until the atomic forces were lower than $0.02 \mathrm{eV} \AA^{-1}$. In many cases, the final position of the molecule lies higher than $3.0 \AA$ from the surface, a too large value to be accurately described within GGA. For this reason, the final configurations have been recalculated including vdW interactions, following the D2-Grimme parametrization ${ }^{43}$ as implemented in VASP. There are other more accurate vdW potentials included in VASP like Tkatchenko-Scheffler, ${ }^{44}$ but they are more expensive computationally. We consider that the $\mathrm{D} 2$ approximation is good enough for the calculations performed here and the final conclusions obtained. For some cases, the incorporation of these long-range forces leads to relevant differences in the structural and energetic output, as we will discuss below.

Finally, the total energy of each molecule adsorbed over the different defects is collected and analyzed. The adsorption energy $E_{\text {ads }}$ is defined as

$$
E_{\text {ads }}=E_{\text {tot }}-E_{\mathrm{Mos}_{2}}-E_{\mathrm{Mol}}
$$

where $E_{\text {tot }}$ is the total energy obtained with the VASP code for the system containing both the $\mathrm{MoS}_{2}$ supercell and the molecule. $E_{\mathrm{Mol}}$ and $E_{\mathrm{MoS}_{2}}$ correspond to the energies of the isolated molecule and the rectangular $\mathrm{MoS}_{2}$ supercell of Fig. 1(a), respectively. In the following sections, we will determine the adsorption energy $E_{\text {ads }}$ and the adsorption distance for all the possible configurations among the most interesting sites on the pristine or defective monolayer and the molecules studied in this work, namely, $\mathrm{CO}_{2}, \mathrm{CO}, \mathrm{O}_{2}, \mathrm{H}_{2} \mathrm{O}, \mathrm{NO}_{2}, \mathrm{NO}, \mathrm{H}_{2}$ and $\mathrm{N}_{2}$. On this basis, we will show that molecules can be either chemisorbed or physisorbed, thus forming either strong or weak bonds with the pristine or defective $\mathrm{MoS}_{2}$ structures, depending on the particular defect-molecule combination considered.

In order to confirm the strong molecule-defect interaction found in certain cases, we performed an electronic analysis for the structures with the highest adsorption energy. On the one hand, the charge transfer between molecules and the substrate upon adsorption was assessed by means of Bader analysis. ${ }^{45}$ In these estimations, a positive charge transfer means that the electrons flow from the defective layer to the molecule. On the other hand, the density of states (DOS) was calculated and subsequently used to simulate the STM image, yielding the fingerprint of each molecule on its most interacting defect. For that purpose, we have followed the non-equilibrium Green's functions formalism developed by Keldysh. ${ }^{46}$ Within this methodology, the equation of the electronic current for an applied voltage $V$ at large distances can be written as $^{47}$

$$
I=\frac{4 \pi e^{2}}{h} \int_{E_{\mathrm{F}}}^{E_{\mathrm{F}}+e V} \operatorname{Tr}\left[T_{\mathrm{TS}} \rho_{\mathrm{SS}}(\omega) T_{\mathrm{ST}} \rho_{\mathrm{TT}}(\omega-e V)\right] \mathrm{d} \omega .
$$

where $\rho_{\mathrm{TT}}$ and $\rho_{\mathrm{SS}}$ are the density matrices associated with the tip and sample subsystems and $T_{\mathrm{TS} / \mathrm{ST}}$ the tip-sample interaction. See ref. 47 for a more detailed explanation. Here, we have used the $\mathrm{W}$ tip previously tested in many works. ${ }^{33,48}$ The $\rho_{\text {TT }}$ and $\rho_{\text {SS }}$ matrices have been obtained using the atomic-like orbital DFT code FIREBALL. ${ }^{49}$ Those localized orbitals are defined by a spatial cutoff radius $\left(r_{\mathrm{c}}\right)^{50}$ whose different values are summarized in Table 1.

Finally, we have complemented the analysis of some potential molecular dissociative processes by estimating the free energy variation $(\Delta G)$ :

$$
\Delta G=\Delta E-T \Delta S
$$

where $\Delta E$ is the energetic change in the process, $T$ is the temperature and $\Delta S$ is the entropy variation. The entropic term is the most difficult part of the equation. It has an electronic contribution that can usually be neglected and a vibrational

Table 1 Cutoff radii (in atomic units) used with the FIREBALL code ${ }^{49}$

\begin{tabular}{llllllll}
\hline Orbital & Mo & $\mathrm{S}$ & $\mathrm{W}$ & $\mathrm{C}$ & $\mathrm{O}^{a}$ & $\mathrm{~N}$ & $\mathrm{H}$ \\
\hline $\mathrm{S}$ & 6.2 & 4.3 & 4.7 & 4.8 & 4.5 & 3.6 & 3.80 \\
$\mathrm{p}$ & 6.2 & 4.7 & 5.2 & 5.4 & 4.5 & 4.1 & - \\
$\mathrm{d}$ & 5.8 & 5.5 & 4.5 & 5.2 & - & 5.2 & -
\end{tabular}

${ }^{a}$ Double basis. 
contribution. Within the DFT methodology we can estimate this last contribution on the defected monolayer using a harmonic approximation and summing up to all the vibrational modes (for more details see the Review). ${ }^{54}$ The molecular entropies at room temperature have been taken from a standard chemical database. ${ }^{55}$

\section{Results and discussion}

In this section, we discuss the results obtained for molecular adsorption and dissociation on both the pristine and defective $\mathrm{MoS}_{2}$ monolayer (containing vacancies and antisite substitutionals). From the energetic results, we will discuss the potential molecular diffusion over the monolayer. As a paradigmatic case, we will firstly address the study of a carbon dioxide molecule $\left(\mathrm{CO}_{2}\right)$ in interaction with a perfect $\mathrm{MoS}_{2}$ monolayer, in order to describe in detail the procedure followed for all the $\mathrm{MoS}_{2}$ structure-molecule combinations analyzed in this work. We will hence discuss the kinds of interactions that may take place when a $\mathrm{CO}_{2}$ molecule is adsorbed over the different defects of the $\mathrm{MoS}_{2}$ monolayer. Subsequently, we will extend this analysis to other molecules. As a conclusion, we will determine the combinations of $\mathrm{MoS}_{2}$ site-molecule that allow the molecular diffusion on the monolayer and the cases that yield the highest energy gain and lead to the formation of a chemical bond. Finally, the molecular dissociation process will be studied for selected cases, revealing the catalytic properties of the defective $\mathrm{MoS}_{2}$ layer.

\subsection{Clean $\mathrm{MoS}_{2}$ and $\mathrm{CO}_{2}$ molecule}

We start by considering the interaction of a $\mathrm{CO}_{2}$ molecule with a pristine $\mathrm{MoS}_{2}$ monolayer. With this first example, the discussion on the nature of the molecule- $\mathrm{MoS}_{2}$ monolayer interaction is initiated. Furthermore, we want to explain how the most stable geometry is determined for a linear molecule. We firstly compare the energy of the molecule adsorbed over different points (on top of a S or Mo atom, over the bridge and hollow sites or on the center of the hexagon) for different molecular orientations. The linear $\mathrm{CO}_{2}$ molecule has five degrees of freedom and consequently it can be adsorbed in many different orientations $^{51}$ The molecule can be placed with the $\mathrm{C}$ or the $\mathrm{O}$ atoms directly positioned over the different sites mentioned before. One example is presented in the frontal view of Fig. 1(a) and the lateral views of the insets of panels (b) and (c). In this case, the molecule shows a parallel configuration with respect to the $\mathrm{S}$ rows of the monolayer. In fact, the figure shows the molecule in two alternative sites, labelled with DFT and VDW. The former case corresponds to the most stable structure found within the DFT approach, i.e. when the dispersive vdW forces are not included. In this structure, the $\mathrm{C}$ atom of the molecule is positioned between the bridge and Mo-top sites at an equilibrium height $\Delta h$ of $3.40 \AA$ (defined as the distance between the lower atom of the molecule and the uppermost $\mathrm{S}$ atoms). Additionally, the molecule was displaced upwards and downwards in each configuration in order to find the most stable position. In all the relaxed structures, the resulting adsorption energies $E_{\text {ads }}$ are very small, between -0.03 and $-0.05 \mathrm{eV}$ per molecule. Using the Local Density Approximation (LDA) functional within the DFT methodology, Yue et al. also obtained small energetic difference on the different sites for a large range of molecules such as $\mathrm{N}_{2}, \mathrm{H}_{2}$, $\mathrm{NO}$ and $\mathrm{NO}_{2} \cdot{ }^{17}$

The small values of the adsorption energy and the large equilibrium distances obtained within the DFT approximation suggest that the vdW interactions should be added to the calculations in order to provide an accurate description of the system. Thus, starting from the DFT-GGA converged geometries, the system is relaxed again including the vdW forces in the simulation, following Grimme's parametrization. ${ }^{43}$ As a result the final geometries have been changed with respect to the ones obtained with GGA. Now, the $\mathrm{CO}_{2}$ molecule is relaxed in a planar configuration at $3.32 \AA$ from the $\mathrm{MoS}_{2}$ monolayer in a slightly shifted position from the center of the hexagon, as shown in Fig. 1(a). The inclusion of the vdW interactions also leads to an increase in the adsorption energy $E_{\text {ads }}$ to $-0.15 \mathrm{eV}$ for that particular case, ranging from -0.13 to $-0.15 \mathrm{eV}$ for all the adsorption sites in good agreement with the value presented by Zhao et al. ${ }^{16}$

In Fig. 1(b) and (c), the evolution of the adsorption energy of the most stable case is presented as a function of the molecular rotation in the $Y Z$ and $X Y$ planes, respectively. In the first case, the molecule reaches a final position perpendicular to the monolayer as the inset in the right part of panel (b) shows. The final energy increases around $0.05 \mathrm{eV}$. In the second case, the molecule is rotated around the $Z$ axis in the $X Y$ plane (see the scheme in the insets of panel (c)) losing less than $0.02 \mathrm{eV}$. We have tried alternative orientations combining both molecular rotations. For example, the molecule can be rotated 45 degrees in the $Y Z$ plane, and later, the adsorption energies can be calculated for different angles in the $X Y$ plane. From all this collection of values, we found that the final most stable structure is the one presented in Fig. 1(a) (labelled with VDW). This result shows that the vdW interaction is optimized when the molecule lies in the $X Y$ plane, parallel to the $S$ rows and slightly displaced from the central point of the hexagon. On the other hand, in panel (d), we have studied the effect of molecular displacement along the $Z$ axis. The energy increases less than $0.05 \mathrm{eV}$ when the molecule is displaced $0.8 \AA$ far away from its most stable configuration. These very small energy differences between the different potential adsorption sites, orientations and heights suggest that the molecule will likely be able to move freely on the surface, without having to overcome any significant diffusion barrier. The small interaction of the $\mathrm{CO}_{2}$ molecule with the pristine monolayer has been previously observed in experimental measurements ${ }^{18}$ and theoretical calculations. ${ }^{31}$

From these preliminary calculations we have shown that the inclusion of vdW forces is mandatory to accurately characterize the position and interaction of a weakly reactive molecule with the $\mathrm{MoS}_{2}$ monolayer. Furthermore, we can deduce that pristine $\mathrm{MoS}_{2}$ is poorly reactive, and hence not suited for catalysis or molecular storage purposes. We will now consider the case of defective $\mathrm{MoS}_{2}$ monolayers, where we have previously proved 
that the reactivity is strongly enhanced, ${ }^{34}$ in order to determine the preferred adsorption sites of the $\mathrm{CO}_{2}$ molecule.

\section{2 $\mathrm{MoS}_{2}$ defects: vacancies and antisites}

Before considering the interaction between $\mathrm{MoS}_{2}$ defects and molecules, we briefly discuss here the formation energy and stability of vacancies and antisites in $\mathrm{MoS}_{2}$. The calculated atomic structures of each defect, presented in Fig. 2, have been discussed in previous works. ${ }^{33,34}$ From (a) to (c), the three clean vacancies are presented: the mono and divacancy of S and the Mo vacancy, respectively. The following panels show the different antisites: one Mo atom in the S vacancy in (d), one and two Mo atoms on the $S$ divacancy in (e) and (f), and finally, one or two $S$ atoms occupying the Mo vacancy in (g) and (h). All these structures have been experimentally observed in a previous work. ${ }^{20}$

Following eqn (1), the formation energy of each defect depends on the energy of each element inside the $\mathrm{MoS}_{2}$ monolayer. While these values can be easily obtained on structures formed by a single element, ${ }^{41}$ the lack of an exact definition leads to an estimation of a reference for each atomic energy. In the present case, the expression of the formation energy has been simplified to eqn (2) in a first step and then, we have considered the energies of each element when they are inside an ideal bulk, namely the bcc structure for Mo and the $\alpha$-orthorhombic structure for $\mathrm{S}$. Consequently, the chemical potential $\mu$ of each element can be initially defined by these bulk energies. It has been previously shown that the chemical potential changes with the experimental conditions, ${ }^{56}$ affecting the formation energies of the defects. For this reason, these energies will be considered as a reference that can give a good trend for the energetic stability of those defects.

The $S$ vacancy presents the lowest formation energy, namely $2.57 \mathrm{eV}$. This value is higher than the energy previously obtained $(2.12 \mathrm{eV})$ due to the slightly different conditions in the calculation. ${ }^{20}$ This means that the $S$ vacancy should be the most frequent defect, in good agreement with the experimental evidence since it is the predominant point defect observed in the STEM measurements on the samples grown by ME and CVD techniques. $^{20}$ This value can be compared with the calculated single vacancy in the $\alpha$-orthorhombic structure of the S-bulk that we have estimated at $0.33 \mathrm{eV}$. This result means that a $S$ atom can be removed from the S-bulk more easily than from a $\mathrm{MoS}_{2}$ monolayer. The formation energy of the $\mathrm{S}$ divacancy is $5.14 \mathrm{eV}$, which is twice the value of the monovacancy, suggesting that the energetic cost of removing one $S$ atom will be independent of the position of the other $S$ vacancies. On the other hand, the formation energy of a Mo vacancy is $7.22 \mathrm{eV}$, again higher than the value presented by Hong and coworkers. ${ }^{20}$ The value is much larger than the formation energy of a vacancy in the Mo perfect bulk $(2.96 \mathrm{eV})$ calculated by Derlet et $a l .{ }^{57}$ The energy difference confirms the great stability of the S-Mo bonds in the monolayer.

The lowest formation energy of the antisites is obtained in the structure formed by a Mo atom occupying the $S$ vacancy $(5.55 \mathrm{eV})$. Interestingly, the value of one single Mo atom in the $\mathrm{S}$ divacancy grows to $7.42 \mathrm{eV}$ and the second Mo atom leads to a much higher value of $10.75 \mathrm{eV}$. In contrast to the clean vacancies, the formation energies are closer to the values calculated by Hong et $a .^{20}$ In that work, these defects were more frequently observed in samples grown using the PVD technique, suggesting a great change in the chemical potential for this experimental technique. On the other hand, when one or two S atoms occupy the Mo vacancy the formation energies take the values $6.35 \mathrm{eV}$ and $9.04 \mathrm{eV}$ respectively, again much higher than the values previously presented in ref. 20 .

Finally, besides quantitative evaluation of the formation energy of the defects (Table 2), our strong argument to ensure

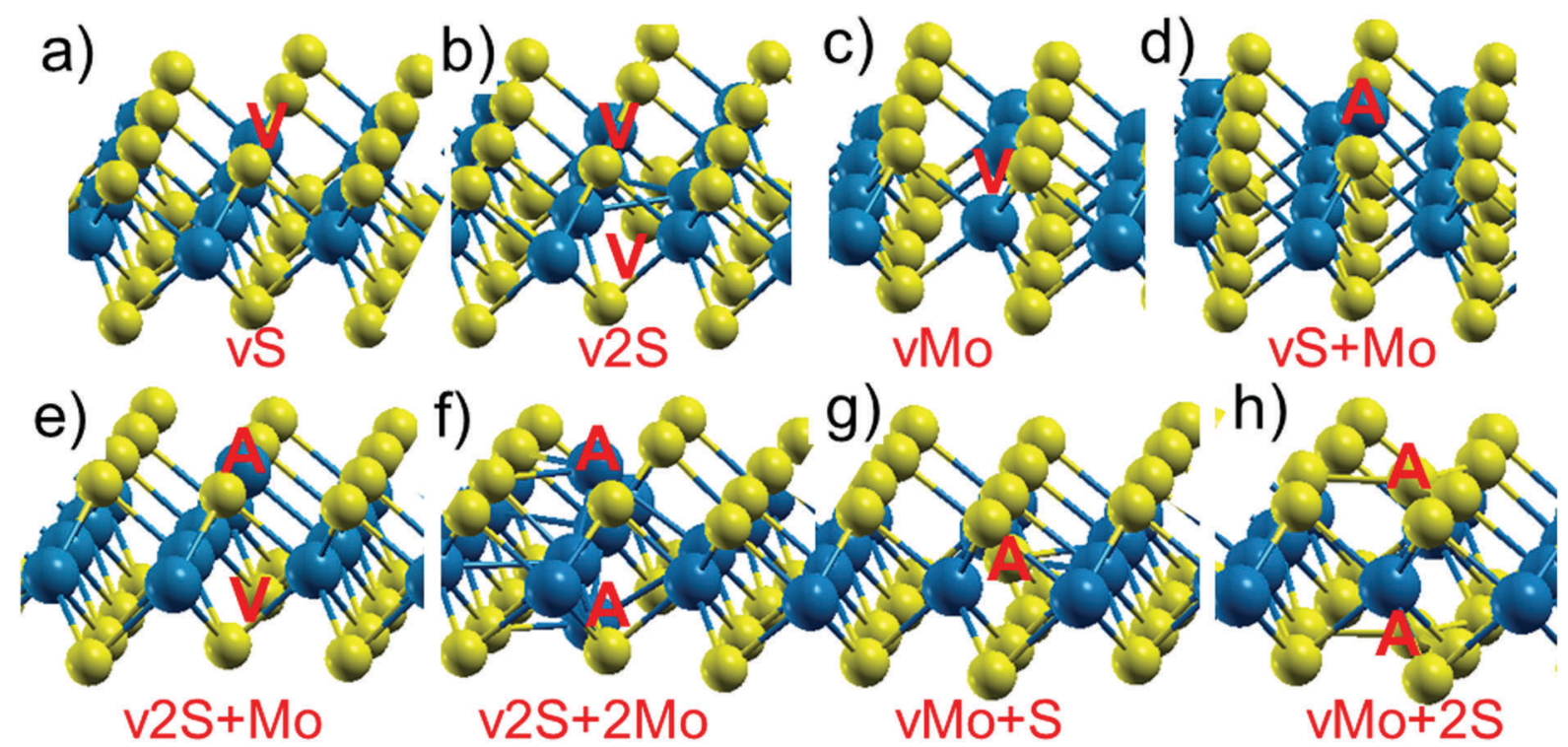

Fig. 2 Lateral view of the ball and stick model of the different defects studied in this work: (a) S vacancy (vS), (b) S divacancy (v2S), (c) Mo vacancy (vMo), (d) a Mo atom occupying the $S$ vacancy $(v S+M o)$, (e) and (f) one ( $(v 2 S+M o)$ and two Mo (v2S + Mo2) atoms occupying the $S$ divacancy, respectively, and $(g)$ and (h) the same for $\mathrm{S}$ atoms in the Mo vacancy (vMo $+\mathrm{S}$ and $\mathrm{vMo}+\mathrm{S} 2$ respectively). The vacancy sites and antisites are indicated with $\mathrm{V}$ and $\mathrm{A}$ labels respectively. 
Table 2 Formation energy in eV of the different defects on the $\mathrm{MoS}_{2}$ monolayer: $\mathrm{S}$ vacancy (vS), Mo vacancy (vMo), S divacancy (v2S), one or two $S$ atoms occupying the Mo vacancy (vMo $+S$ and vMo + S2) and one or two Mo atoms in a S mono- and divacancy (vS + Mo, v2S + Mo and v2S + Mo2, respectively). The formation energies have been calculated using the energy in the perfect Mo or S bulk

\begin{tabular}{lllccl}
\hline & vS & v2S & vMo & vS + Mo & v2S + Mo \\
\hline$E_{\mathrm{f}}(\mathrm{eV})$ & 2.57 & 5.14 & 7.22 & 5.55 & 7.42 \\
\hline \multirow{2}{*}{$E_{\mathrm{f}}(\mathrm{eV})$} & \multirow{2}{*}{$\mathrm{v} 2 \mathrm{~S}+\mathrm{Mo} 2$} & \multicolumn{2}{c}{$\mathrm{vMo}+\mathrm{S}$} & $\mathrm{vMo}+\mathrm{S} 2$ \\
\hline
\end{tabular}

the defect stability is based on our ab initio MD calculations. All those simulations have been performed at room temperature for all the defects analyzed previously. Our results confirm the stability in all the cases. A movie of the atomic motion in the defective structure formed by two Mo atoms occupying the $\mathrm{S}$ divacancy during 30 fs is shown in ESI. $\dagger$ The atoms oscillate around the most stable structure presented in Fig. 2.

\section{3 $\mathrm{CO}_{2}$ adsorption on $\mathrm{MoS}_{2}$ defects}

In this section we have followed the procedure described in the previous section to analyze the interaction of $\mathrm{CO}_{2}$ with a defective $\mathrm{MoS}_{2}$ layer. The resulting adsorption energies and distances are summarized in Tables 3 and 4, respectively, for the most stable molecular configurations obtained over each defect. All the values presented in both the tables are obtained including the vdW interactions in the calculation. The corresponding atomic structures for four selected defects, namely Mo and $\mathrm{S}$ vacancies, and one and two $\mathrm{S}$ substitutionals in a

Table 3 Adsorption energies (eV) of the most stable adsorption sites for each molecule

\begin{tabular}{llllllll}
\hline Molecules & $\mathrm{CO}_{2}$ & $\mathrm{CO}$ & $\mathrm{O}_{2}$ & $\mathrm{H}_{2} \mathrm{O}$ & $\mathrm{NO}$ & $\mathrm{N}_{2}$ & $\mathrm{H}_{2}$ \\
\hline Pristine & -0.15 & -0.09 & -0.11 & -0.14 & -0.15 & -0.09 & -0.06 \\
vS & -0.16 & -0.14 & -3.04 & -0.25 & -2.88 & -0.12 & -0.08 \\
vS + Mo & -0.59 & -1.95 & -5.12 & -1.05 & -3.15 & -1.54 & -1.14 \\
vMo & -0.17 & -0.11 & -2.49 & -0.16 & -0.22 & -0.10 & -0.06 \\
vMo + S & -0.14 & -0.10 & -0.61 & -0.19 & -0.90 & -0.09 & -0.06 \\
vMo + S2 & -0.14 & -0.10 & -0.55 & -0.08 & -0.12 & -0.09 & -0.05 \\
v2S & -0.16 & -0.14 & -9.35 & -0.24 & -2.93 & -0.11 & -0.08 \\
v2S + Mo & -0.41 & -1.04 & -4.12 & -1.28 & -3.10 & -0.09 & -0.52 \\
v2S + Mo2 & -1.19 & -1.84 & -4.88 & -1.63 & -4.21 & -1.16 & -0.78
\end{tabular}

Table 4 Equilibrium distance $(\AA)$ of the most stable adsorption sites for each molecule. The sites labelled with * correspond to the dissociative cases

\begin{tabular}{llllllll}
\hline Molecules & $\mathrm{CO}_{2}$ & $\mathrm{CO}$ & $\mathrm{O}_{2}$ & $\mathrm{H}_{2} \mathrm{O}$ & $\mathrm{NO}$ & $\mathrm{N}_{2}$ & $\mathrm{H}_{2}$ \\
\hline Pristine & 3.32 & 3.36 & 2.78 & 2.37 & 2.73 & 3.53 & 2.75 \\
vS & 2.97 & 2.09 & $-^{*}$ & 0.85 & 1.01 & 2.90 & 1.90 \\
vS + Mo & 2.36 & 2.00 & 2.01 & 2.29 & 1.82 & 1.98 & $-^{*}$ \\
vMo & 3.30 & 3.00 & $-^{*}$ & 2.37 & 0.95 & 3.53 & 3.30 \\
vMo + S & 3.50 & 3.25 & 1.66 & 2.37 & 1.92 & 3.50 & 2.83 \\
vMo + S2 & 3.51 & 1.92 & 2.52 & 2.70 & 2.51 & 3.00 & 3.23 \\
v2S & 2.95 & 2.30 & $-^{*}$ & 1.05 & 1.13 & 3.04 & 2.60 \\
v2S + Mo & 2.31 & 2.20 & 2.01 & 2.21 & 1.82 & 2.10 & 1.89 \\
v2S + Mo2 & 2.24 & 2.05 & 2.01 & 2.21 & 1.81 & 2.05 & 1.88
\end{tabular}

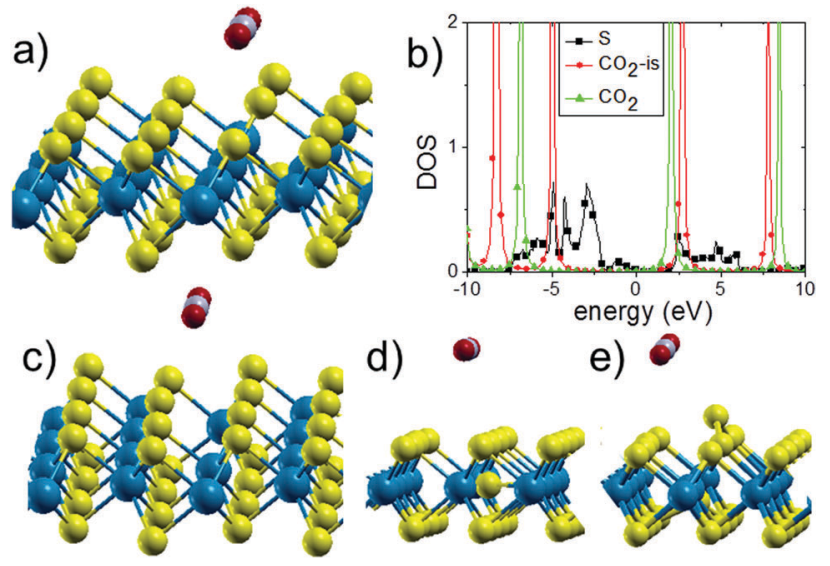

Fig. 3 Equilibrium geometry of a $\mathrm{CO}_{2}$ molecule physisorbed on a $\mathrm{MoS}_{2}$ monolayer with (a) S vacancy, (c) Mo vacancy, (d) and (e) one and two $S$ substitutional atoms occupying a Mo vacancy; (b) DOS of a $S$ atom far away from the vacancy (black squares), the $\mathrm{CO}_{2}$ placed over the $S$ vacancy (green triangles) and the isolated molecule (red circles). The Fermi energy is fixed at $0 \mathrm{eV}$.

Mo vacancy, are shown in Fig. 3. In panel (a), the $\mathrm{CO}_{2}$ molecule is adsorbed over a $\mathrm{S}$ vacancy. Despite the presence of the dangling bonds of the unsaturated atoms around the vacancy, which suggests the possibility of a strong chemical bond with the molecule, we found that it remains still physisorbed at a distance of $2.97 \AA$. This is confirmed by the DOS presented in Fig. 3(b) for a $\mathrm{S}$ atom far away from the vacancy (black squares) and the $\mathrm{CO}_{2}$ over the monolayer (green triangles). Together with them, the DOS of the isolated molecule (red circles) is shown (the Fermi level is fixed at 0 energy in all the cases). The molecular states are only slightly displaced from the isolated case due to the weak interaction. Although the adsorption distance in the S vacancy case is $0.35 \AA$ lower than that for the most stable structure on the pristine monolayer, the adsorption energy only grows from -0.15 to $-0.16 \mathrm{eV}$. This small value confirms the vdW interaction. Notice that both energy and molecular distance remain almost unchanged on a S di-vacancy. Over the Mo vacancy shown in Fig. 3(c), the molecule is adsorbed at almost the same distance as on the pristine layer $(3.3 \AA)$, but the energy grows to $-0.17 \mathrm{eV}$, which is almost the same value as over the $\mathrm{S}$ vacancy. In all the studied cases, the differences between these energies and the values obtained in the perfect $\mathrm{MoS}_{2}$ are again very small, and consequently, the $\mathrm{CO}_{2}$ molecules will also be able to diffuse on the vacancies of the monolayer. Our energetic calculations are in good agreement with previous simulations in the case of the Mo vacancy, but they are in disagreement with the stronger interaction obtained over the $S$ vacancy calculated with an atomic-like orbital code. ${ }^{31}$ We have tried to approach the molecule to the vacancy by hand, but after the relaxation it was detached at vdW distances. Probably the difference in the basis set leads to an increase in the molecular attraction by the dangling bonds around the vacancy.

Similar adsorption energies have been found when the molecule is adsorbed over other defects (all the values are summarized in Table 3). For example, the defects formed by 
one or two S atoms occupying a Mo vacancy present an adsorption energy of $-0.14 \mathrm{eV}$. Regarding the molecule-surface distances, the molecule is located around $3.50 \AA$ above the $\mathrm{S}$ atoms of the monolayer. In the first case, the $\mathrm{CO}_{2}$ molecule maintains the planar configuration over the vacancy site. It is detached from the substitutional atom (see Fig. 3(d)), which in turn is displaced with respect to the original position of the missing Mo atom (a deeper explanation of the atomic configuration of this defect can be found in ref. 33). On the other hand, in the second case, the molecule loses the planar configuration, being slightly tilted and displaced from its original position over the substitutional $\mathrm{S}$ atoms as shown in the ball and stick model of Fig. 3(e). In this configuration, the monolayer is affected by the interaction with the molecule even though the $\mathrm{CO}_{2}$ is placed far away from the $\mathrm{MoS}_{2}$. The topmost substitutional $\mathrm{S}$ atom has been shifted to a higher position, losing the bond with one of the neighbouring atoms. The structure of this defect can be compared with the one previously obtained in a clean environment. ${ }^{33}$

The situation changes significantly when metallic defects are considered, namely the cases of one substitutional Mo atom in a S mono-vacancy and one or two Mo atoms occupying a $S$ di-vacancy. These sites present an important contribution of the DOS at the Fermi level (see ref. 33 for details). In Fig. 4(a) and (b), the adsorption of the $\mathrm{CO}_{2}$ molecule over the first and third defects mentioned is shown. Additionally, the real-space electronic density integrated between -0.5 and $0.5 \mathrm{eV}$ is represented in the figures. In these two cases, the molecule- $\mathrm{MoS}_{2}$ distance has been estimated around 2.40 and $2.24 \AA$, respectively, and a chemical bond is formed with the Mo substitutional atom. A similar result is obtained for the other metallic defect, one Mo atom inside a S di-vacancy, where the molecule is adsorbed at $2.37 \AA$. As a consequence of this molecule- $\mathrm{MoS}_{2}$ distance

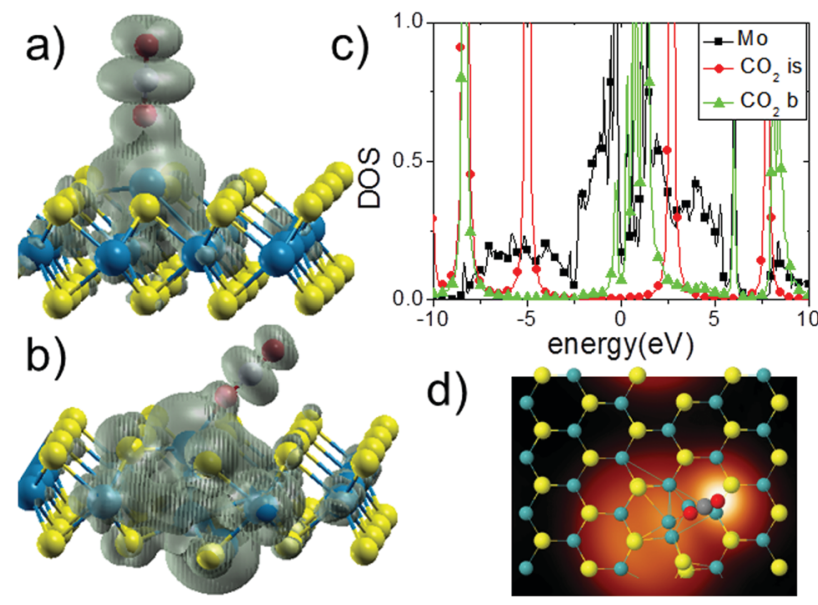

Fig. 4 Real-space electronic density integrated between -0.5 and $0.5 \mathrm{eV}$ with the equilibrium geometry of a $\mathrm{CO}_{2}$ molecule chemisorbed on a $\mathrm{MoS}_{2}$ monolayer with (a) one Mo substitutional atom in a S mono-vacancy and (b) two Mo substitutional atoms occupying a S di-vacancy. (c) The DOS are shown for the substitutional Mo atom (black squares) and $\mathrm{CO}_{2}$ molecule (green triangles) of the (b) structure and the isolated molecule (red circles). The Fermi energy is fixed at $0 \mathrm{eV}$. (d) Corresponding simulated STM image at $V=-1.0 \mathrm{~V}$ (the image was created using the WSxM software). ${ }^{53}$ reduction, a stronger bond has been formed between one oxygen from the molecule and the substitutional Mo atom. This stronger interaction is reflected in an increase of the adsorption energy and in the great change in the DOS (Fig. 4(c)), which is reflected in the bond formed in the real-space electronic density.

The most interacting defect is the $\mathrm{S}$ di-vacancy occupied by two Mo substitutional atoms with a value of $-1.19 \mathrm{eV}$ (see Table 3). The two other substitutional cases present a weaker interaction but still more important (more than twice bigger) than the one obtained for the pristine sites and the previous defects. On the other hand, the DOS of the molecule bonded with the Mo atom (green triangles and black squares respectively) is shown in Fig. 4(c). Compared to the DOS of the isolated molecule, the originally well-defined molecular states have changed and some new peaks have appeared around the Fermi level, in coincidence with the Mo atom states bonded with the $\mathrm{CO}_{2}$. This behaviour is different from that over the $\mathrm{S}$ vacancy where only state displacement is obtained. Now, the Mo-O bond is correlated with the common peaks at $-8.1 \mathrm{eV},+5.95 \mathrm{eV}$, $+8.3 \mathrm{eV}$ and the states around the Fermi level as explained before. ${ }^{58}$ We have applied the Bader analysis in order to estimate the charge transfer. Interestingly, even though there is an important DOS reorganization, the charge transfer established between the molecule and the defect is rather low (0.04 electrons) as shown in Table 5 . Notice that the charge transfer in the other metallic defects is quite similar, reflecting the great electronic stability of the $\mathrm{CO}_{2}$ molecule.

Consequently, we can conclude that the molecule is trapped by the defect and could thus be imaged by STM. Fig. 4(d) shows the simulated image for a bias of $-1.0 \mathrm{~V}$. The occupied states of the molecule close to the Fermi level produce a bright protrusion around the highest $\mathrm{O}$ atom of the molecule. The interaction between the molecule and the substitutional Mo atom blurs the well-localized maximum over the clean defect (see the image in ref. 33).

As a first remark, we can observe that the adsorption energies are significantly stronger for the metallic defects than for the other defects or adsorption sites of pristine $\mathrm{MoS}_{2}$. For the sake of comparison, we can contrast our results with those obtained for $\mathrm{CO}_{2}$ adsorption on graphene, where the physisorption energy is slightly smaller but the chemisorption energy in defective graphene is more important. ${ }^{25}$

Taking into account these energetic values, the molecule is expected to diffuse along the pristine monolayer and also when only single vacancies or $\mathrm{S}$ substitutionals are encountered during its motion. On the other hand, the $\mathrm{CO}_{2}$ molecule will probably be stabilised close to the metallic defects, reducing drastically its mobility. As in the interaction with a Si tip

Table 5 Charge transfer (e) obtained in the adsorption of the different molecules in the metallic defects

\begin{tabular}{lllllll}
\hline Molecules & $\mathrm{CO}_{2}$ & $\mathrm{CO}$ & $\mathrm{O}_{2}$ & $\mathrm{H}_{2} \mathrm{O}$ & $\mathrm{NO}$ & $\mathrm{N}_{2}$ \\
\hline vS + Mo & 0.02 & 0.30 & 0.84 & 0.09 & 0.53 & 0.28 \\
v2S + Mo & 0.02 & 0.18 & 0.83 & 0.09 & 0.51 & 0.29 \\
v2S + Mo2 & 0.04 & 0.28 & 0.88 & 0.10 & 0.50 & 0.35
\end{tabular}


previously analyzed, ${ }^{34}$ the enhanced interaction between the molecule and the metallic defects can be explained by means of a semiconductor-metal junction model, the role of the semiconducting material being played by the $\mathrm{CO}_{2}$ molecule.

Finally, we can also observe that the orientation of the adsorbed molecule with respect to the $\mathrm{MoS}_{2}$ surface is strongly related to the metallic/semiconductor character of the defect. Indeed, for the semiconducting defects or perfect layer, the molecule remains parallel (or almost parallel) to the surface due to the weak vdW interactions. On the other hand, for metallic defects, the molecule-layer interaction increases, leading to the rotation of the molecule to favor a stronger Mo-O bond in the metal-semiconductor junction.

\subsection{Adsorbing other molecules on the $\mathrm{MoS}_{2}$ layer}

A similar procedure was followed for other molecules studied in this work, namely carbon oxide (CO), nitrogen mono- (NO) and dioxide $\left(\mathrm{NO}_{2}\right)$, water $\left(\mathrm{H}_{2} \mathrm{O}\right)$, and the homonuclear diatomic molecules like dioxygen $\left(\mathrm{O}_{2}\right)$, dinitrogen $\left(\mathrm{N}_{2}\right)$ and dihydrogen $\left(\mathrm{H}_{2}\right)$. All these molecules may be important for testing the trapping or catalytic activity of the different sites and defects of the $\mathrm{MoS}_{2}$ monolayer. For this reason, we have carefully analyzed the most stable configurations and adsorption energies of those molecules on the pristine and defective $\mathrm{MoS}_{2}$ monolayer following the procedure previously explained for the $\mathrm{CO}_{2}$ molecule. All the results for the most stable geometries of each molecule on the different $\mathrm{MoS}_{2}$ sites are summarized in Tables 3 and 4 .

The cases with largest adsorption energy for the $\mathrm{CO}$ and NO molecules are represented in Fig. 5. Considering the case of a $\mathrm{CO}$ molecule adsorbed on a S mono-vacancy occupied by a Mo atom (Fig. 5(a)), the adsorption behavior is apparently similar to that of $\mathrm{CO}_{2}$, except that, in this case, the molecule is most favorably bonded to the Mo atom of the defect through the carbon atom with a greater adsorption energy of $-1.95 \mathrm{eV}$ at a shorter equilibrium distance of $2.00 \AA$. The molecule can also bind through the oxygen atom but the final structure presents a smaller interacting energy of $-0.73 \mathrm{eV}$. This result was expected since we have previously found that a $\mathrm{CO}$ molecule tends to bond through the $\mathrm{C}$ atom to a metallic Au tip. ${ }^{47}$ Thus, our result confirms that the two adsorption configurations are absolutely non-equivalent. The analysis of the DOS presented in Fig. 5(b) and its real-space projection of panel (a) are in agreement with the large interaction. The DOS of the bonded molecule (green triangles) shows the bonding and anti-bonding states around the Fermi level in clear contrast with the isolated case (red circles). There is a clear correlation between the peaks of $\mathrm{CO}$ and Mo atoms at $-7.5 \mathrm{eV}$, around the Fermi level and at $+4.6 \mathrm{eV}$, showing the strong bond established between them. In this case, the larger adsorption energy is accompanied by a larger charge transfer $\left(\begin{array}{lll}0.30 & e\end{array}\right)$ than in the $\mathrm{CO}_{2}$ molecule, reflecting the fact that a stronger bond has been established. Finally, the simulated STM image for a $-1.0 \mathrm{~V}$ bias (Fig. 5(c)) shows a maximum over the molecule due to a $\mathrm{p}_{z}$ contribution of the CO (not shown in the figure).

A similar final structure, adsorption energy and charge transfer is found for the defect formed by two Mo atoms occupying the
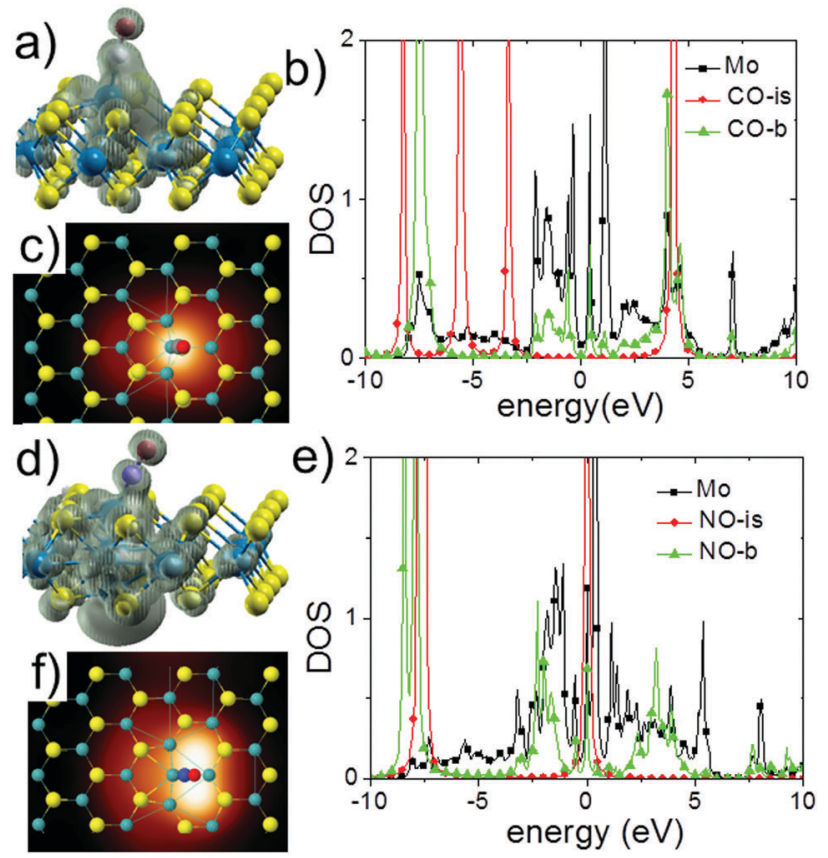

Fig. 5 Real-space electronic density integrated between -0.5 and $0.5 \mathrm{eV}$ with the equilibrium geometry of different adsorbed molecules on a defective $\mathrm{MOS}_{2}$ monolayer like (a) CO and (d) NO. In (b) and (e) the DOS of the Mo atom (black square) bonded to each molecule (green triangles) are shown together with the DOS of the isolated molecule for $\mathrm{CO}$ and NO, respectively. The Fermi energy is fixed at $0 \mathrm{eV}$. The STM image simulated at $V=-1.0 \mathrm{~V}$ is presented in (c) and (f) for $\mathrm{CO}$ and $\mathrm{NO}$.

$\mathrm{S}$ di-vacancy $\left(E_{\mathrm{ads}}=-1.84 \mathrm{eV}\right)$. From the collection of values presented in the tables, we confirm again that the molecule prefers to bond on metallic sites, while on the semiconducting sites the adsorption energy is much lower (between $-0.09 \mathrm{eV}$ and $-0.14 \mathrm{eV}$ ), allowing the molecular diffusion on the $\mathrm{MoS}_{2}$ layer. The low interaction with the $\mathrm{S}$ vacancy is in contradiction with the chemisorption recently proposed. ${ }^{29}$ We have tried to force the formation of a O-Mo or C-Mo bond, approaching the molecule to distances close to the vacancy, but the molecule is finally detached at the end of the simulation. In contrast to the $\mathrm{CO}_{2}$ case, the adsorption value over the pristine monolayer differs from the value presented by Zhao et al., where a value around $-0.075 \mathrm{eV}$ was obtained within the same approximations. ${ }^{16}$ This means that in the present work a more stable structure has been found.

The following molecule to be analyzed is the nitrogen oxide. Due to its composition (formed by one $\mathrm{O}$ atom and another element from the second row of the period table), it can be expected to behave like the $\mathrm{CO}$ molecule, but the extra electron introduced by the $\mathrm{N}$ atom changes critically the results at certain points. The most important difference comes from its enhanced interaction with the S mono- and di-vacancies $(-2.88$ and $-2.93 \mathrm{eV})$ and the substitutional S atom $(-0.90 \mathrm{eV})$, which were only weakly bonded to the $\mathrm{CO}$ molecule through $\mathrm{vdW}$ interactions. These values suggest that the NO molecule will not likely diffuse over the S vacancies, remaining bonded to the Mo atoms of the defect. The molecular chemisorption 
was previously found in a similar theoretical simulation. ${ }^{29}$ With respect to the adsorption sites on the pristine monolayer, the molecule presents an interaction similar to the previous molecules $\left(E_{\mathrm{ads}}=-0.15 \mathrm{eV}\right)$ in agreement with previous calculations. ${ }^{16}$ This is reflected in its closer adsorption position $(2.73 \AA)$. Finally, in agreement with the previously considered molecules, the most reactive sites are again the three metallic cases. The atomic configuration of the most attractive site is shown in Fig. 5(d) and its corresponding adsorption energy has been estimated at $-4.21 \mathrm{eV}$ with an equilibrium distance of $1.81 \AA$ A. Notice that the molecule is bonded to the Mo atom through the $\mathrm{N}$ atom, instead of the oxygen. The energy difference with respect to that situation is $1.78 \mathrm{eV}$, i.e. approximately $1 \mathrm{eV}$ more than in the CO case. The DOS of the molecule, both isolated (green triangles) and bonded to the substitutional Mo atom (black squares), is shown in Fig. 5(e). Again, the interaction implies a great change in the molecular DOS compared to the isolated case (red circles). In this structure, there is a larger charge transfer $(0.53 e)$ associated to the larger adsorption energy due to the higher reactivity of NO. Some common peaks of the Mo atom bonded to the molecule at around $-2.5 \mathrm{eV}$, at the Fermi level and around $+2.5 \mathrm{eV}$ can also be found. In contrast to CO, the simulated STM image, shown in Fig. 5(f), presents a maximum formed by two lobes due to the strong weight of the $\mathrm{p}_{y}$-orbital of the $\mathrm{O}$ atom in the DOS.

We now turn to the water molecule. In this case, we have one additional degree of freedom due to the non-linear character of the molecule. ${ }^{51}$ Fig. 6(a) shows the three initial orientations used in this work. As explained previously with the $\mathrm{CO}_{2}$, the molecule is adsorbed with the $\mathrm{O}$ or $\mathrm{H}$ atoms directly positioned over each site and, in this case, the molecule can rotate around the three axes. In Fig. 6(b), the most attractive structure is shown for a water molecule. Again, the three substitutional Mo defects present a much higher adsorption energy, ranging from

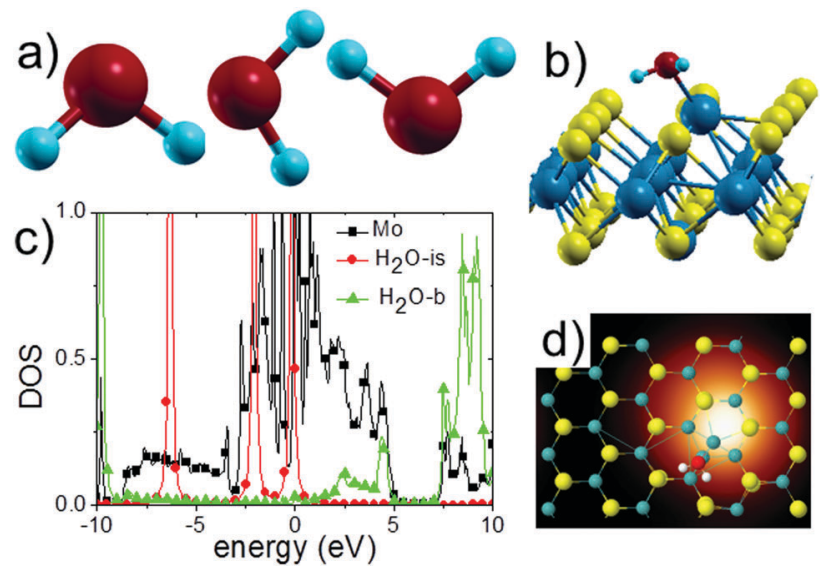

Fig. 6 (a) The three orientations of the water molecule studied in this work are presented; (b) equilibrium geometry of a water molecule adsorbed on a defect formed by two substitutional Mo atoms on a $\mathrm{S}$ divacancy on a $\mathrm{MoS}_{2}$ monolayer. (c) DOS of the Mo atom (black square) bonded to the water molecule (green triangles) and DOS of the isolated molecule (red circles). The Fermi energy is fixed at $0 \mathrm{eV}$. The STM image simulated at $V=-1.0 \mathrm{~V}$ is presented in (d).
-1.63 to $-1.05 \mathrm{eV}$, than the other defects and the pristine layer (from -0.08 to $-0.24 \mathrm{eV}$ ). Again we have found a more attractive structure than in Zhao's work $(-0.16 \mathrm{eV} v s .-0.08 \mathrm{eV}) .{ }^{16}$ All the values are summarized in Table 3. Taking these values into account, we can conclude that water should easily diffuse over a defective monolayer, with only the metallic substitutional defects containing Mo atoms being able to trap the molecule, as also happens with most of the previously analyzed molecules. Now, $\mathrm{H}_{2} \mathrm{O}$ is bonded to the Mo atom through the oxygen and placed 2.21 A far away from it. In this case, the Mo-O bond has changed the molecular DOS displacing the states far away from the Fermi level to $-10.0 \mathrm{eV},+5.0 \mathrm{eV}$ and $+10 \mathrm{eV}$ and only a very small contribution is observed around the Fermi level as shown in Fig. 6(b). This leads to a small charge transfer of $0.1 e$ between the $\mathrm{CO}_{2}$ and $\mathrm{CO}$ cases as happened with the adsorption energy. The change of the DOS is also reflected in the simulated STM image of Fig. 6(c) that now has the maximum slightly displaced from the Mo atom bonded to the molecule. The water molecule is closer to the topmost $\mathrm{S}$ atoms over other defects, such as Mo and S vacancy (with 2.37 and $0.87 \AA$ respective heights), but the real distance to the atoms around is still estimated as more than $3.00 \AA$ and consequently, no bond is formed with the atoms from the monolayer.

Finally, we would like to study three different homonuclear diatomic molecules, namely $\mathrm{N}_{2}, \mathrm{O}_{2}$ and $\mathrm{H}_{2}$. Again, for the $\mathrm{N}_{2}$ case, the most interacting defect with $-1.54 \mathrm{eV}$ is one substitutional Mo atom in a S mono-vacancy. The atomic structure shown Fig. 7(a) reflects the fact that the molecule is tilted from the axis perpendicular to the layer, $1.98 \AA$ above the Mo atom. Again the metal-semiconductor interface is favored with a charge transfer from the metallic defect to the molecule ranging from 0.25 to $0.34 e$ in the most attractive case (see Table 5). On the other hand, the semiconducting defects and the pristine monolayer present adsorption energies (ranging from $-0.12 \mathrm{eV}$ over the $\mathrm{S}$ vacancy to $-0.09 \mathrm{eV}$ on the pristine monolayer in good agreement with the value presented in ref. 16) and equilibrium distances characteristic of vdW interaction (from 2.90 to $3.53 \AA$ ). These results are similar to the values obtained in previous molecules. Due to the Mo-N bond, some molecular states arise close to the Fermi level, clearly affecting the DOS (see Fig. 7(b)).

The second homonuclear diatomic molecule is $\mathrm{O}_{2}$. The ball and stick model displayed in Fig. 7(c) shows the structure with the molecule adsorbed on the metallic defect formed by a Mo atom occupying a $S$ vacancy (the most reactive case of the three substitutional cases involving new Mo atoms). Now the adsorption energy is much higher than before $(-5.12 \mathrm{eV})$ and the molecule is placed at a distance of $1.87 \AA$ with respect to the substitutional Mo atom. It is important to notice that in this case both atoms of the molecule are bonded to the Mo substitutional, which justifies the important energetic gain. From the relaxed structure of the figure, we can conclude that the $\mathrm{O}$ atoms are bonded to each other, maintaining the molecular structure. In fact the atomic distance between both $\mathrm{O}$ atoms is estimated around $1.45 \AA$, only $0.21 \AA$ larger than for the isolated molecule. The calculated DOS of the isolated (red circles) and bonded molecule (green triangles) and the substitutional Mo atom 

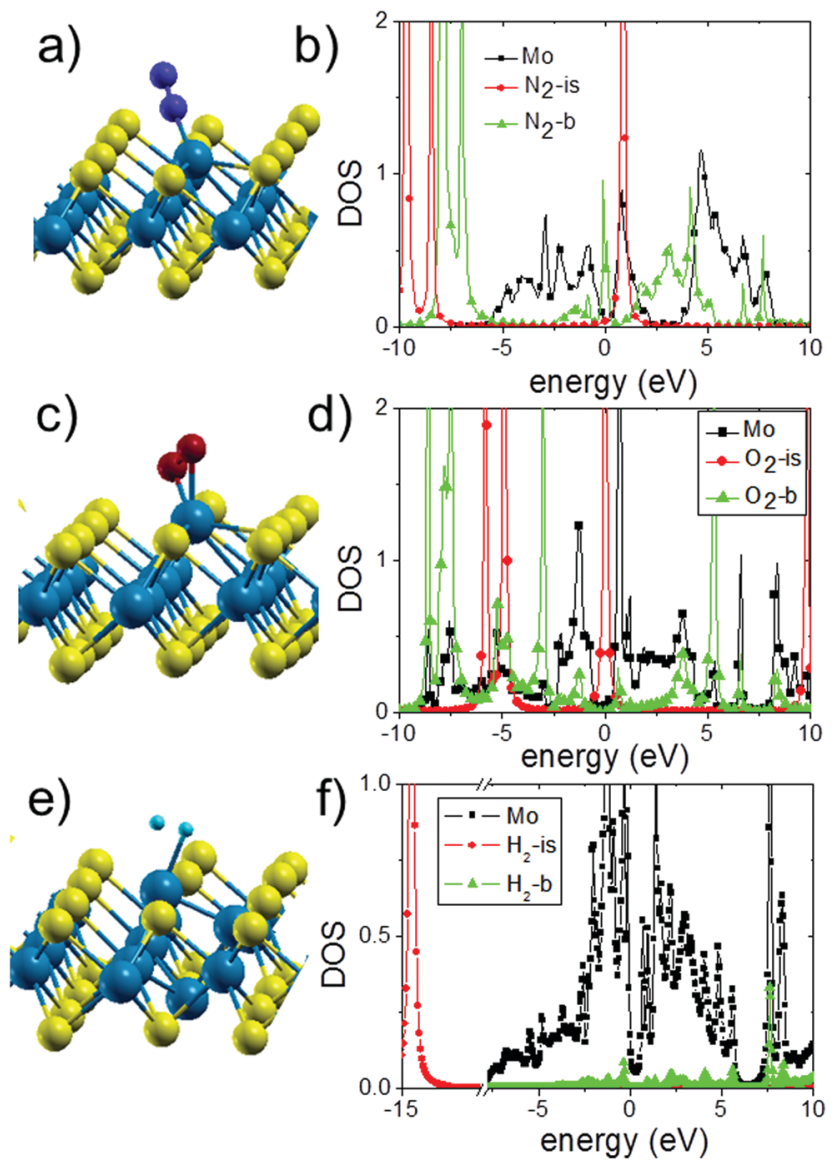

Fig. 7 Equilibrium geometry of different adsorbed molecules on a defective $\mathrm{MoS}_{2}$ monolayer (a) $\mathrm{N}_{2}$, (c) $\mathrm{O}_{2}$ and (e) $\mathrm{H}_{2}$. In (b), (d) and (f) the DOS of the Mo atom (black square) bonded to each molecule (green triangles) are shown together with the DOS of the isolated molecule for $\mathrm{N}_{2}, \mathrm{O}_{2}$ and $\mathrm{H}_{2}$, respectively. The Fermi energy is fixed at $0 \mathrm{eV}$.

(black squares) are shown in Fig. 7(d). In the isolated form, the molecule presents a state at the Fermi level related with its unpaired electrons. As happened with the previous molecules, we can observe different common peaks of the molecule and Mo atom, directly linked to the bonds established between them. The peaks at $-1.0 \mathrm{eV},+0.7 \mathrm{eV}$ and at deeper energies like $-7.5 \mathrm{eV}$ or $-8.3 \mathrm{eV}$ represent a good illustration. Following the trend of the other molecules, the larger attraction leads to a higher charge transfer. In this case, the value is $0.88 e$, confirming the higher reactivity of this molecule.

Again, we obtain similar atomic geometries for the other two metallic defects including Mo substitutional atoms, but now the calculated adsorption energies are slightly smaller $(-4.12$ and $-4.88 \mathrm{eV})$ for one and two Mo atoms in a $\mathrm{S}$ di-vacancy, respectively. In contrast to previous molecules, $\mathrm{O}_{2}$ strongly interacts with the dangling bonds of other semiconducting defects. For example, the molecule is bonded to the three Mo atoms surrounding a $\mathrm{S}$ monovacancy as previously reported by $\mathrm{Li}$ et $a l^{29}$ It is adsorbed perpendicularly to the monolayer, occupying the empty space left by the missing $\mathrm{S}$ atom (the geometry is not shown in the figure). The $\mathrm{O}-\mathrm{O}$ bond has been enlarged to $1.36 \AA$, a slightly lower value than in the previous defect. Two very recent works have demonstrated both theoretically and experimentally that the $\mathrm{O}_{2}$ can be dissociated in the $\mathrm{S}$ vacancy. ${ }^{27,28}$ We have calculated the structure formed by one $\mathrm{O}$ atom in the vacancy and a second atom bonded to a $\mathrm{S}$ atom close to the vacancy as proposed by Akdim et al. This alternative geometry was found to be $2.75 \mathrm{eV}$ more stable than the one previously obtained in our simulations, suggesting that probably the $\mathrm{O}_{2}$ molecule could be dissociated in the S vacancy as Qi et al. demonstrated. ${ }^{27}$

On the other hand, the $\mathrm{O}_{2}$ molecule is detached over the substitutional $\mathrm{S}$ atoms, presenting a much lower adsorption energy ( -0.61 and $-0.55 \mathrm{eV}$ for one and two atoms respectively) than the substitutional Mo cases, but still much larger than the pristine layer $(-0.11 \mathrm{eV})$. We will discuss the Mo vacancy and $\mathrm{S}$ divacancy cases later.

Comparing the results of both homonuclear diatomic molecules $\left(\mathrm{N}_{2}\right.$ and $\left.\mathrm{O}_{2}\right)$, it should be noticed that neither the most stable configuration nor its corresponding adsorption energy value is equivalent. This expected difference can be explained by the different reactivity of both molecules: the $\mathrm{O}_{2}$ molecule is highly reactive due to its unpaired electrons while $\mathrm{N}_{2}$ is less reactive as revealed by the adsorption energies. The preference for the metallic defects and the vdW interaction established with the pristine monolayer and the substitutional $\mathrm{S}$ atoms can be found as coincident points. Our results for the $\mathrm{O}_{2}$ case imply that it may diffuse only over the pristine monolayer and the substitutional S defects, but on the contrary, it will be highly attracted and trapped by the other defects.

The last diatomic molecule analyzed in this work is $\mathrm{H}_{2}$. The study of its adsorption is of particular importance since it might be exploited for hydrogen storage. The molecular structure with the highest molecule-substrate interaction is shown in Fig. $7(\mathrm{e})$ and its corresponding DOS and STM image are presented in (e) and (f). The isolated $\mathrm{H}_{2}$ molecule presents only two peaks at -14.65 and $+14.65 \mathrm{eV}$ (only the first one is shown in Fig. 7(f)). When the molecule is bonded to the Mo atom, a non-negligible (but small) contribution to the DOS appears for energies close to the Fermi level. In this structure, one of the $\mathrm{H}$ atoms forming the molecule is bonded to the highest Mo atom that is occupying the S di-vacancy. Interestingly, the distance between both $\mathrm{H}$ atoms is slightly larger than in the isolated molecule ( $0.83 \AA$ vs. $0.75 \AA$ ), suggesting that the molecule is still formed but also that it might dissociate at a given temperature.

Indeed, dissociation does occur when the $\mathrm{H}_{2}$ is adsorbed onto another metallic defect (one substitutional Mo in a $\mathrm{S}$ monovacancy), as shown in Fig. 9(a). This ball and stick model presents a geometry where both $\mathrm{H}$ atoms are connected to the Mo atom, increasing the energy of the system, and where the original $\mathrm{H}-\mathrm{H}$ bond is completely broken. The energy in this case is estimated at $-1.14 \mathrm{eV}$, close to the value obtained for one single $\mathrm{H}$ atom adsorbed on the same site $(-0.93 \mathrm{eV})$, showing that the $\mathrm{H}$-Mo interaction changes when two $\mathrm{H}$-atoms are bonded to the same molybdenum. On the other hand, when the $\mathrm{H}_{2}$ molecule is placed over the pristine monolayer or the semiconducting defects, the low adsorption energies (between -0.05 and $-0.08 \mathrm{meV}$ ) at the standard vdW distances mean that 


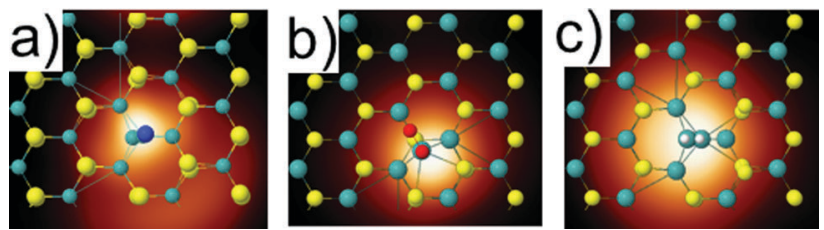

Fig. 8 Simulated STM image at voltage $V=-1.0 \mathrm{~V}$ for (a) $\mathrm{N}_{2}$, (b) $\mathrm{O}_{2}$ and (c) $\mathrm{H}_{2}$ on their corresponding most attractive defect.

the molecule will likely diffuse over the substrate. We have studied the adsorption of one single $\mathrm{H}$ atom over the $\mathrm{MoS}_{2}$ monolayer and we have found that it can be bonded to both $\mathrm{S}$ and Mo atoms in a similar way to the $\mathrm{C}$ atoms in the graphene monolayer. ${ }^{52}$ The adsorption energies are estimated as $-1.52 \mathrm{eV}$ and $-1.12 \mathrm{eV}$, respectively. This last value is larger than the value presented before for a bond with a Mo atom in an antisite. The H-S distance takes the value of $1.4 \AA$, very similar to $1.35 \AA$, the $\mathrm{H}-\mathrm{S}$ distance calculated in a $\mathrm{H}_{2} \mathrm{~S}$ molecule.

Interestingly, the most stable structures of the three homonuclear diatomic molecules lead to apparently similar STM images as presented in Fig. 8. Panel (a) shows the $\mathrm{N}_{2}$ case where a bright protrusion is obtained over the molecule due to the molecular states found close to the Fermi level (see Fig. 7(b)). In the $\mathrm{O}_{2}$ case, the DOS distributed around the Fermi level presents a dominant contribution of the $\mathrm{p}_{y}$ orbital as well as an important contribution of the $\mathrm{p}_{z}$ orbital. The $\mathrm{p}_{y}$ contribution slightly displaces the bright spot from the substitutional Mo and the topmost $\mathrm{O}$ atoms, as shown in panel (b). Finally, STM image in the $\mathrm{H}_{2}$ case is basically the same as the one obtained for the clean defect, i.e. without the molecule when the maximum is placed over the Mo substitutional atom as we previously demonstrated. ${ }^{33}$ This result is directly linked to the very small DOS contribution of the $\mathrm{H}$ atoms around the Fermi level. Consequently, these three molecules could only be distinguished by comparing the electronic current on the maximum point.

Summarizing the results presented up to now, we can conclude that the small molecules tend to diffuse over the pristine monolayer and, in most cases, also over the defective monolayer, provided that the defects have a semiconducting character. Only the $\mathrm{O}_{2}$ and $\mathrm{NO}$ molecules form strong bonds with the dangling bonds around the vacancies. In all the other cases, both molecule and monolayer are weakly interacting through vdW forces. On the other hand, the molecules can be trapped and chemisorbed over the metallic defects to form stronger bonds with the $\mathrm{MoS}_{2}$ atoms. Our results are supported by the close examination of the DOS, which shows important contributions around the Fermi level due to the molecule-Mo bonding and the analysis of the charge transfer which is generally larger for the cases with higher adsorption energy. In addition, the strong interaction with the molecule does affect the STM image over these metallic defects, presenting each molecule a characteristic fingerprint.

Our results hence confirm that in the molecule-defective $\mathrm{MoS}_{2}$ interaction, the formation of a metal-semiconductor junction is clearly favored, as previously suggested for an AFM tip. ${ }^{34}$ For selected cases, dissociation can occur as it happens for the $\mathrm{H}_{2}$ case, thus conferring a catalytic nature to some defects of the $\mathrm{MoS}_{2}$ layer. To further explore this possibility we will extend this study in the following section.

\subsection{Molecular dissociation on $\mathrm{MoS}_{2}$ vacancies}

We finally turn to the study of the dissociation process that might take place for certain molecules when adsorbed onto some specific defects on the $\mathrm{MoS}_{2}$ monolayer. This process represents a promising way to enhance the catalytic properties of defective $\mathrm{MoS}_{2}$. One case has already been mentioned in the previous section, namely the $\mathrm{H}_{2}$ molecule adsorbed over the Mo substitutional on the $\mathrm{S}$ vacancy shown in Fig. 9(a). The second case, displayed in panel (b), corresponds to the $\mathrm{O}_{2}$ molecule dissociated in the Mo vacancy. In this structure, both $\mathrm{O}$ atoms stay bonded to the dangling bonds of different atoms around the vacancy. Interestingly, the adsorption energy shows that this case is less interacting than the case of the same molecule adsorbed over the metallic defects: $-2.49 \mathrm{eV} v s$. $-5.12 \mathrm{eV}$ (see Table 3), which means that the molecule would prefer to bind with the Mo substitutional atom instead of dissociating over the Mo vacancy. On the contrary, our calculations show that the $\mathrm{O}_{2}$ molecule is as well dissociated in the S divacancy (see Table 4) leading to a great energetic benefit of $-9.35 \mathrm{eV}$. In this structure, the original $\mathrm{S}$ atoms have been replaced by the $\mathrm{O}$ atoms in a slightly displaced position on the pristine monolayer. The structures presented here are not surprising as it was previously demonstrated both experimentally and theoretically that $\mathrm{O}_{2}$ molecules can be dissociated on defective $\mathrm{MoS}_{2}$ layers. ${ }^{27,28}$ At any case, in order to avoid this potential dissociation, it will be necessary to cover or saturate the $\mathrm{S}$ and Mo vacancies with other atoms.

The last molecule studied in this work is nitrogen dioxide, $\mathrm{NO}_{2}$. The molecule shows a stronger interaction than the molecules studied in the previous sections, between $-0.28 \mathrm{eV}$ with the pristine monolayer and the sulphur vacancies and $-0.46 \mathrm{eV}$ on the S-substitutional structures. These results are in agreement with the calculations presented before. ${ }^{17}$ As happened with the other molecules, the lowest value of the adsorption energy

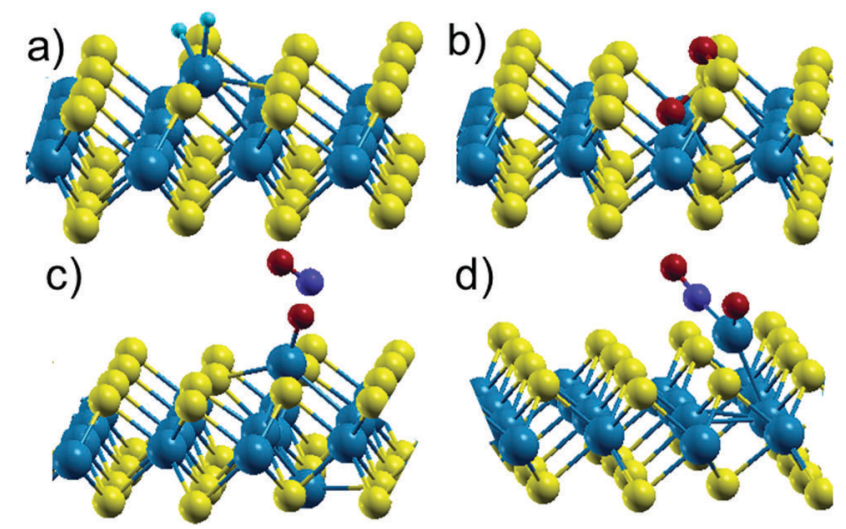

Fig. 9 Atomic representation of the dissociative configurations of different molecules on a defective $\mathrm{MoS}_{2}$ monolayer: (a) $\mathrm{H}_{2}$ dissociated on the substitutional Mo atom occupying a $\mathrm{S}$ vacancy, (b) $\mathrm{O}_{2}$ molecule dissociated in a Mo vacancy, (c) $\mathrm{NO}_{2}$ over two Mo atoms occupying the S-divacancy and (d) $\mathrm{NO}_{2}$ over a Mo substitutional atom on a $\mathrm{S}$ vacancy. 
is obtained when the molecule is placed on the metallic defects, but, interestingly, in these cases the $\mathrm{NO}_{2}$ is always dissociated. In Fig. 9(c) and (d) we show two different ways of dissociation. In the first case, with two Mo atoms occupying the sulphur di-vacancy, the molecule breaks into one $\mathrm{O}$ atom and a $\mathrm{NO}$ molecule. The NO molecule is desorbed and only the $\mathrm{O}$ atom remains bonded with the Mo atom. In the second case, the $\mathrm{NO}_{2}$ molecule is adsorbed over the Mo substitutional atom on the $\mathrm{S}$ di-vacancy. On this site, although the same separation into an isolated $\mathrm{O}$ and a NO molecule takes place, both parts remain bonded to the Mo atom. This structure corresponds to the case with stronger adsorption energy, probably due to the second bond created with the NO molecule. It is important to note that the corresponding adsorption energy $(-4.23 \mathrm{eV})$ is almost the same as that obtained for a NO molecule over the defect formed by two Mo atoms occupying the S di-vacancy $(-4.21 \mathrm{eV})$, showing that the Mo-NO bond is not the same in both situations. This is somehow similar to the case involving two $\mathrm{H}$ atoms bonded to a substitutional Mo atom.

The mechanism underlying all those dissociation processes is related to the interaction with the atoms with unpaired states around the defects. We have already demonstrated in a previous work ${ }^{33}$ that the defect sites lead to the emergence of several very reactive states in the original gap of $\mathrm{MoS}_{2}$. Indeed, when a molecule is adsorbed on the $\mathrm{MoS}_{2}$ surface, it will diffuse on the pristine part of the surface, due to the weak vdW interactions. At some point, the molecule will be electrostatically attracted by the defect. Obviously the Mo metallic states on the metallic antisites and around the vacancies are electronically rich, and will react with the most electronegative atom of the molecule. This specific attraction reflects the O-Mo interaction for the $\mathrm{O}_{2}$ dissociation on the S divacancy and Mo vacancy as well as for the $\mathrm{NO}_{2}$ molecule on the metallic defects. Then, when an O-Mo bond is formed, it is energetically more stable than the intramolecular bonds, which, added to the strong electronic exchange between the two atoms, yields the molecular dissociation (the missing electrons for the $\mathrm{O}$ atom are given by the Mo atom). In that respect, molecules will be able to lose one oxygen atom through a connection to a Mo atom. This process is obviously favored for the case of the Mo substituted $S$ vacancy, due to the prominence of the Mo atom on top of the surface, but it is obtained as well for the Mo atoms close to the $\mathrm{S}$ or Mo vacancies.

These results show the potential catalytic properties of the defective $\mathrm{MoS}_{2}$ monolayer. However, all of the calculations presented in this work have been performed at $0 \mathrm{~K}$ and, consequently, the dissociation processes occur only in the cases where there is no energetic barrier to overcome. Nevertheless, a temperature increase may induce dissociation even in the presence of significant energy barriers. Using the $a b$ initio-MD technique implemented in the FIREBALL code, we have analyzed the stability of the previous molecules in their corresponding more attractive sites at room temperature. During the period of simulation (30 ps), the molecules $\mathrm{CO}, \mathrm{NO}, \mathrm{N}_{2}, \mathrm{H}_{2} \mathrm{O}$ and $\mathrm{O}_{2}$ oscillate around the most stable configuration. On the contrary, the $\mathrm{CO}_{2}$ molecule is dissociated in our simulation as shown in
Movie 2 of ESI. $\dagger$ The formation of the Mo-O bond weakened the $\mathrm{C}-\mathrm{O}$ bond of the $\mathrm{CO}_{2}$ molecule. The molecular vibrations, produced when the thermal effects are included, lead to the break of such $\mathrm{C}-\mathrm{O}$ bond. As a consequence, a $\mathrm{CO}$ molecule remains in the system. Due to the thermal agitation, this new molecule is able to approach the Mo atom and even the dissociated $\mathrm{O}$ atom, but no permanent bond is formed between them. Finally, the molecule is desorbed to standard vdW distances. This result suggests that a higher temperature is required for the dissociation of the other molecules.

On the other hand, combining the energies presented in Table 3, other alternative processes can be proposed for the molecular dissociation. As previously we found $\mathrm{O}_{2}$ dissociation on the $\mathrm{S}$ and Mo vacancies, we will focus our attention on their potential catalytic processes for other molecules: $\mathrm{CO}_{2} \rightarrow \mathrm{CO}+\mathrm{O}$, $\mathrm{CO}_{2} \rightarrow \mathrm{C}+\mathrm{O}_{2}, \mathrm{NO}_{2} \rightarrow \mathrm{NO}+\mathrm{O}, \mathrm{NO}_{2} \rightarrow \mathrm{N}+\mathrm{O}_{2}$ or $\mathrm{H}_{2} \mathrm{O} \rightarrow \mathrm{H}_{2}+\mathrm{O}$. For this purpose, we have performed additional simulations where each vacancy is filled with a $\mathrm{C}, \mathrm{O}$ or $\mathrm{N}$ atom. Thus, one atom of the original $\mathrm{CO}_{2}, \mathrm{NO}_{2}$ or $\mathrm{H}_{2} \mathrm{O}$ will stay bonded occupying the free site in the vacancy, while the dissociated molecule will be able to diffuse over the $\mathrm{MoS}_{2}$ layer.

We can thus compare the energies of the initial and final steps of the process. The initial step corresponds to the original molecule adsorbed on the vacancy $(E(\mathrm{vMo} / \mathrm{S}+\mathrm{MOL}))$, the energy values of lines vMo and vS in Table 3 , while the final step is found when one atom occupies the vacancy $(E(\mathrm{vMo} / \mathrm{S}+\mathrm{C} / \mathrm{O} / \mathrm{N}))$ and the residual molecule diffuses to a perfect area of the $\mathrm{MoS}_{2}$ $\left(E\left(\mathrm{MoS}_{2}+\mathrm{MOL}\right)\right)$. In order to perform the energetic comparison, we need to sum up the energies of the two rectangular unit cells previously calculated including the defects and molecules. In our initial step, we need to add the energy of a second unit cell of exactly the same size for the isolated pristine monolayer, $E\left(\mathrm{MoS}_{2}\right)$. The dissociative process will be possible if the energy of the final step is lower than that of the initial step:

$E(\mathrm{vMo} / \mathrm{S}+\mathrm{MOL})+E\left(\mathrm{MoS}_{2}\right)>E(\mathrm{vMo} / \mathrm{S}+\mathrm{C} / \mathrm{O} / \mathrm{N})+E\left(\mathrm{MoS}_{2}+\mathrm{MOL}\right)$

Using the condition of eqn (5), we have found that none of the potential dissociative processes mentioned before is energetically favourable on the Mo vacancy, but some of them might take place over a $S$ vacancy (see the values in Table 6). Interestingly, the three cases with a negative balance imply the adsorption of one $\mathrm{O}$ atom occupying the $\mathrm{S}$ vacancy. This is

Table 6 Energy/free energy $(\mathrm{eV})$ difference between the initial and final states in alternative dissociative processes

\begin{tabular}{|c|c|c|c|}
\hline Molecules & $\mathrm{CO}_{2}$ & $\mathrm{H}_{2} \mathrm{O}$ & $\mathrm{NO}_{2}$ \\
\hline $\mathrm{vS}-\mathrm{C}+\mathrm{MoS}_{2}-\mathrm{O} 2$ & 5.76 & - & - \\
\hline $\mathrm{vS}-\mathrm{O}+\mathrm{MoS}_{2}-\mathrm{CO}$ & $-0.77 /-0.72$ & - & - \\
\hline$v S-O+\mathrm{MoS}_{2}-\mathrm{H}_{2}$ & - & $-1.32 /-1.14$ & - \\
\hline $\mathrm{vS}-\mathrm{O}+\mathrm{MoS}_{2}-\mathrm{NO}$ & - & - & $-2.84 /-2.73$ \\
\hline $\mathrm{vS}-\mathrm{N}+\mathrm{MoS}_{2}-\mathrm{O} 2$ & - & - & 0.09 \\
\hline vMo-C $+\mathrm{MoS}_{2}-\mathrm{O} 2$ & 6.16 & - & - \\
\hline $\mathrm{vMo}-\mathrm{O}+\mathrm{MoS}_{2}-\mathrm{CO}$ & 2.65 & - & - \\
\hline $\mathrm{vMo}-\mathrm{O}+\mathrm{MoS}_{2}-\mathrm{H}_{2}$ & - & 1.94 & - \\
\hline $\mathrm{vMo}-\mathrm{O}+\mathrm{MoS}_{2}-\mathrm{NO}$ & - & - & 2.54 \\
\hline vMo-N $+\mathrm{MoS}_{2}-\mathrm{O} 2$ & - & - & 1.36 \\
\hline
\end{tabular}


understandable because the $\mathrm{O}$ atom has the same electronic structure as the sulphur, which explains the great energetic benefit in the $\mathrm{O}$ adsorption on the $\mathrm{S}$ vacancy. The $\mathrm{CO}_{2}$ dissociation on the $\mathrm{C}$ vacancies of graphene was previously proposed theoretically by Cabrera-SanFelix. ${ }^{25}$ The dissociation of other molecules such as $\mathrm{H}_{2} \mathrm{O}$ has been previously reported on $\mathrm{TiO}_{2},{ }^{23}$ or the $\mathrm{NO}_{2}$ molecule on $\mathrm{ZnO}^{24}$ Consequently, we can expect that, at a certain temperature, these processes could occur, hence improving the catalytic properties of the $\mathrm{MoS}_{2}$ monolayer. In order to test this possibility, we have performed $a b$ initio MD simulations at room temperature for a relatively long period of time ( $30 \mathrm{ps}$ ) for these three structures taking into account different initial configurations. We have firstly validated the MD-FIREBALL simulations recalculating the structures obtained with VASP. We have confirmed that in the three cases the molecules remain physisorbed over the $S$ vacancy. The adsorption energy obtained with this code is around $-0.30 \mathrm{eV}$ because the interactions are overestimated for the LDA approximation used for the exchange and correlation potential. Our MD calculations show that in some cases, the molecules diffuse over the monolayer, moving far away from the $\mathrm{S}$ vacancy (this result is in agreement with our speculations based on the adsorption energies of the static calculations), while in other cases, the $\mathrm{CO}_{2}$ and $\mathrm{NO}_{2}$ molecules were dissociated at room temperature. See both dissociation processes in Movies 3 and 4 of ESI. $\dagger$ Our results are in good agreement with previous calculations. Li et al. obtained a small energy barrier of $-0.21 \mathrm{eV}$ for the $\mathrm{NO}_{2}$ dissociation in the $\mathrm{S}$ vacancy. ${ }^{29}$

The missing $\mathrm{S}$ creates Mo dangling bonds, ${ }^{33}$ but the Mo atom is located much deeper "inside" the $\mathrm{MoS}_{2}$ structure, complicating the accessibility of the adsorbed molecule. Thus, the Mo-O connection can require an extra energy. In that respect, the connection will depend on the repulsive barrier to be overcome for the $\mathrm{O}$ atom in interaction with the surrounding surface atoms. This last aspect is probably related with the spatial orientation of the molecule. For example, the $\mathrm{CO}_{2}$ molecule is oscillating and rotating around the $S$ vacancy in the initial steps of Movie 3 (ESI $\dagger$ ) until it takes an almost perpendicular orientation. At this moment, the lowest $\mathrm{O}$ atom creates a bond with the Mo atoms closer to the vacancy site. After that, the $\mathrm{O}-\mathrm{C}$ bond is weakened and several steps further, it is finally broken. At the end, the resulting $\mathrm{CO}$ molecule is detached to standard vdW distances. In the case of the $\mathrm{NO}_{2}$ molecule, it reaches the perpendicular geometry in fewer steps. Again the energy barrier can be overcome and one oxygen atom is approached to the Mo sublayer, establishing a connection with the inner Mo atoms in the $\mathrm{S}$ vacancy. After several steps, the $\mathrm{N}-\mathrm{O}$ bond is broken leading to the molecular dissociation. This process is shown in Movie 4 (ESI $\dagger$ ). On the other hand, the $\mathrm{H}_{2} \mathrm{O}$ molecule cannot go through this potential barrier. Therefore, even though the molecule is electrostatically attracted and remains located in the neighborhood of the defect, it cannot be dissociated. In this case, the mechanism implies the formation of Mo-O bonds but additionally two $\mathrm{H}-\mathrm{O}$ bonds should be broken. It seems that the energy barrier could be much larger. Of course, a higher temperature might lead to the final dissociation by overcoming this potential energy barrier.
To summarize, the molecular dissociation processes originate from the metallic dangling bonds of the $\mathrm{MoS}_{2}$ defects associated with the Mo atom, and the strong electronegativity of the $\mathrm{O}$ atoms of the molecule. For a future work, it would be interesting to compare the different dissociation mechanisms associated with molecules composed of other atomic species than oxygen.

We have complemented the $a b$ initio MD simulations, estimating the free energy variation as explained in the Theory section for these three interesting cases. The values for room temperature are included in Table 6. The inclusion of the entropy disorder (at least at room temperature) maintains the final configurations with a lower free energy. These results seem to confirm the energetic result and the potential dissociation obtained on the MD simulations with the $\mathrm{CO}_{2}$ and $\mathrm{NO}_{2}$ molecules. The unsuccessful dissociation of the $\mathrm{H}_{2} \mathrm{O}$ molecules suggests that a larger energy barrier has to be overcome and probably a higher temperature is required. In order to estimate such a barrier or temperature a more detailed analysis should be performed in future works.

A final procedure is linked to the so-called hydrodesulfurization processes. Based on the potential dissociation of the $\mathrm{H}_{2} \mathrm{~S}$ molecule on the S-vacancy, the empty hole can be covered by the $\mathrm{S}$ atom followed by $\mathrm{H}_{2}$ desorption. Eqn (5) can be simplified in this case, as $E(\operatorname{vacS}+\mathrm{S})=E$ (pristine), and we need to compare only the energy of the system with a $\mathrm{H}_{2} \mathrm{~S}$ molecule adsorbed close to the $\mathrm{S}$ vacancy and the energy of the pristine monolayer with a $\mathrm{H}_{2}$ molecule. The second geometry is more stable by $2.55 \mathrm{eV}$. This great energetic difference seems to favor the reaction. In fact, the $\mathrm{H}_{2} \mathrm{~S}$ molecule prefers by $0.04 \mathrm{eV}$ to occupy the $\mathrm{S}$ vacancy leading to a pristine monolayer with an $\mathrm{S}$ atom bonded to two $\mathrm{H}$ atoms. Therefore, the complete process will start with the approach and implantation of the molecule in the $\mathrm{S}$ vacancy, and finally, an energy barrier should be overcome to form the $\mathrm{H}_{2}$ molecule that will be subsequently desorbed. The opposite process implies the formation of an $\mathrm{S}$ vacancy when two $\mathrm{H}$ atoms can be bonded to a $\mathrm{S}$ atom in the pristine monolayer, forming a $\mathrm{H}_{2} \mathrm{~S}$ molecule inside the material. After that, the molecule is desorbed after an energetic addition, leaving a $S$ vacancy behind. This process has been previously presented on the edges of $\mathrm{MoS}_{2}$ clusters and surfaces rich in $\mathrm{S}$ atoms. ${ }^{5-61}$

\section{Conclusions}

In conclusion, we have performed DFT + vdW simulations to study the reactivity of a defective $\mathrm{MoS}_{2}$ monolayer with common small inorganic molecules. Our results show that the molecules are weakly bonded through vdW interactions over the pristine monolayer and the semiconducting defects (Mo and S vacancies and S substitutional cases), thus allowing their diffusion over the $\mathrm{MoS}_{2}$ substrate. Only in some specific cases such as $\mathrm{O}_{2}$ or NO on the $\mathrm{S}$ vacancy, the molecule strongly interacts with these kinds of defects. On the other hand, the molecules are highly attracted by the metallic defects formed by Mo atoms occupying the $\mathrm{S}$ mono- or di-vacancy. In those cases, a stronger bond can be formed between the Mo substitutional 
atoms and the molecule. This leads to a notable increase of the adsorption energy accompanied by a larger charge transfer, hence suggesting that the molecules will be trapped in the defect, prohibiting their diffusion over the monolayer.

Moreover, we show that in some cases, the molecule is dissociated over the metallic defects even at $0 \mathrm{~K}$ (the temperature at which our DFT + vdW simulations were performed), suggesting the potential catalytic properties of these sites. To further explore this issue we have analyzed the energetic balance of other alternative dissociative processes. The results show that molecules such as $\mathrm{CO}_{2}$ or $\mathrm{NO}_{2}$ can be dissociated on the $\mathrm{S}$ vacancy at room temperature, leaving one $\mathrm{O}$ atom occupying the empty space. Our work thus opens the way to the possibility of tuning the catalytic properties of $\mathrm{MoS}_{2}$ by the controlled creation of specific defects in the $\mathrm{MoS}_{2}$ monolayer in order to favor adsorption for storage, gas detection or dissociation.

\section{Acknowledgements}

The authors acknowledge the computer resources by the Spanish Supercomputing Network (RES), project QCM-2016-1-0024, and the Alhambra supercomputer facilities at University of Granada. C. G. acknowledges funding by the Junta de Andalucía and the European Commission under the Co-funding of the 7th Framework Program in the People Program through the Andalucia Talent Hub program. Research by BB is supported by MINECO (Spain) under the Ramon y Cajal program.

\section{References}

1 K. Novoselov, A. K. Geim, S. V. Morozov, D. Jiang, Y. Zhang, S. V. Dubonos, I. V. Grigorieva and A. A. Firsov, Science, 2004, 306, 666.

2 K. Watanabe, T. Taniguchi and H. Kanda, Nat. Mater., 2004, 3, 404.

3 R. Zboril, F. Karlicky, A. B. Bourlinos, T. A. Steriotis, A. K. Stubos, V. Georgakilas, F. Safarova, D. Jancik, C. Trapalis and M. Otyepka, Small, 2010, 6, 2885-2891.

4 N. Myoung, K. Seo, S. J. Lee and G. Ihm, ACS Nano, 2013, 7, 7021.

5 K. F. Mak, C. Lee, J. Hone, J. Shan and T. F. Heinz, Phys. Rev. Lett., 2010, 105, 136805.

6 A. M. Jones, et al., Nat. Nanotechnol., 2013, 8, 634-638.

7 Y. Zhang, T.-R. Chang, B. Zhou, Y.-T. Cui, H. Yan, Z. Liu, F. Schmitt, J. Lee, R. Moore, Y. Chen, H. Lin, H.-T. Jeng, S.-K. Mo, Z. Hussain, A. Bansil and Z.-X. Shen, Nat. Nanotechnol., 2013, 9, 111.

8 Y. Wu, Y. Lin, A. A. Bol, K. A. Jenkins, F. Xia, D. B. Farmer, Y. Zhu and P. Avouris, Nature, 2011, 472, 72-78.

9 M. Pumera, Z. Soferb and A. Ambrosi, J. Mater. Chem. A, 2014, 2, 8981.

10 R. Ionescu, A. George, I. Ruiz, Z. Favors, Z. Mutlu, C. Liu, K. Ahmed, R. Wu, J. S. Jeong, L. Zavala, K. A. Mkhoyan, M. Ozkanacd and S. Ozkan, Chem. Commun., 2014, 50, 11226-11229.
11 F. K. Perkins, A. L. Friedman, E. Cobas, P. M. Campbell, G. G. Jernigan and B. T. Jonker, Nano Lett., 2013, 13(2), 668-673.

12 B. Cho, M. G. Hahm, M. Choi, J. Yoon, A. R. Kim, Y.-J. Lee, S.-G. Park, J.-D. Kwon, C. S. Kim, M. Song, Y. Jeong, K.-S. Nam, S. Lee, T. J. Yoo, C. G. Kang, B. H. Lee, H. C. Ko, P. M. Ajayan and D.-H. Kim, Sci. Rep., 2015, 5, 8052.

13 B. Liu, L. Chen, G. Liu, A. N. Abbas, M. Fathi and C. Zhou, ACS Nano, 2014, 8, 5304.

14 H. Li, Z. Yin, Q. He, H. Li, X. Huang, G. Lu, D. W. H. Fam, A. I. Y. Tok, Q. Zhang and H. Zhang, Small, 2012, 8, 63.

15 Q. He, Z. Zeng, Z. Yin, H. Li, S. Wu, X. Huang and H. Zhang, Small, 2012, 8, 2994-2999.

16 S. Zhao, J. Xue and W. Kang, Chem. Phys. Lett., 2014, 595-596, 35.

17 Q. Yue, Z. Shao, S. Chang and J. Li, Nanoscale Res. Lett., 2013, 8, 425.

18 Y. Qi, Q. Xu, Y. Wang, B. Yan, Y. Ren and Z. Chen, ACS Nano, 2016, 10, 2903-2909.

19 W. Zhou, X. Zou, S. Najmaei, Z. Liu, Y. Shi, J. Kong, J. Lou, P. M. Ajayan, B. I. Yakobson and J. C. Idrobo, Nano Lett., 2013, 13, 2615-2622.

20 J. Hong, Z. Hu, M. Probert, K. Li, D. Lv, X. Yang, L. Gu, N. Mao, Q. Feng and L. Xie, et al., Nat. Commun., 2015, 6, 6293.

21 H. Li, C. Tsai, A. L. Koh, L. Cai, A. W. Contryman, A. H. Fragapane, J. Zhao, H. S. Han, H. C. Manoharan, F. Abild-Pedersen, J. K. Nørskov and X. Zheng, Nat. Mater., 2016, 15, 48.

22 M. Makarova, Y. Okawa and M. Aono, J. Phys. Chem. C, 2012, 116, 22411.

23 O. Bikondoa, C. L. Pang, R. Ithnin, C. A. Muryn, H. Onishi and G. Thornton, Nat. Mater., 2006, 5, 189-192.

24 N. Dupont-Paulovsky and F. Carlap, J. Catal., 1977, 46, 426-430.

25 P. Cabrera-Sanfelix, J. Phys. Chem. A, 2009, 113, 493.

26 H. H. Güurel, V. O. Özçelik and S. Ciraci, J. Phys. Chem. C, 2014, 118, 27574-27582.

27 L. Qi, Y. Wang, L. Shen and Y. Wu, Appl. Phys. Lett., 2016, 108, 063103.

28 B. Akdim, R. Pachter and S. Mou, Nanotechnology, 2016, 27, 185701.

29 H. Li, M. Huang and G. Cao, Phys. Chem. Chem. Phys., 2016, 18, 15110-15117.

30 M. P. K. Sahoo, J. Wang, Y. Zhang, T. Shimada and T. Kitamura, J. Phys. Chem. C, 2016, 120, 14113-14121.

31 N. Yu, L. Wang, M. Li, X. Sun, T. Hou and Y. Li, Phys. Chem. Chem. Phys., 2015, 17, 11700-11704.

32 S.-Y. Cho, S. J. Kim, Y. Lee, J.-S. Kim, W.-B. Jung, H.-W. Yoo, J. Kim and H.-T. Jun, ACS Nano, 2015, 9, 9314-9321.

33 C. González, B. Biel and Y. J. Dappe, Nanotechnology, 2016, 27, 105702.

34 C. González, Y. J. Dappe and B. Biel, J. Phys. Chem. C, 2016, 120, 17115-17126.

35 G. Kresse and J. Hafner, Phys. Rev. B: Condens. Matter Mater. Phys., 1993, 47, R558; G. Kresse and J. J. Furthmuller, Phys. 
Rev. B: Condens. Matter Mater. Phys., 1996, 54, 11169; G. Kresse and D. Joubert, Phys. Rev. B: Condens. Matter Mater. Phys., 1999, 59, 1758.

36 P. E. Blöchl, Phys. Rev. B: Condens. Matter Mater. Phys., 1994, 50, 17953.

37 J. P. Perdew, K. Burke and M. Ernzerhof, Phys. Rev. Lett., 1996, 77, 3865.

38 N. Wakabayashi, H. G. Smith and R. M. Nicklow, Phys. Rev. B: Solid State, 1975, 12, 659.

39 J.-Y. Noh, H. Kim and Y. S. Kim, Phys. Rev. B: Condens. Matter Mater. Phys., 2014, 89, 205417.

40 H.-P. Komsa and A. V. Krasheninnikov, Phys. Rev. B: Condens. Matter Mater. Phys., 2015, 91, 125304.

41 C. González, D. Fernández-Pello, M. A. Cerdeira, S. L. Palacios and R. Iglesias, Modell. Simul. Mater. Sci. Eng., 2014, 22, 035019.

42 H. J. Monkhorst and J. D. Pack, Phys. Rev. B: Solid State, 1976, 13, 5188.

43 S. Grimme, J. Comput. Chem., 2006, 27, 1787-1799.

44 A. Tkatchenko and M. Scheffler, Phys. Rev. Lett., 2009, 102, 073005.

45 W. Tang, E. Sanville and G. Henkelman, J. Phys.: Condens. Matter, 2009, 21, 084204.

46 J. M. Blanco, C. González, P. Jelínek, J. Ortega, F. Flores and R. Pérez, Phys. Rev. B: Condens. Matter Mater. Phys., 2004, 70, 085405.

47 C. González, E. Abad, Y. J. Dappe and J. C. Cuevas, Nanotechnology, 2016, 27, 105201.

48 C. Sánchez-Sánchez, C. González, P. Jelinek, J. Méndez, P. L. de Andres, J. A. Martín-Gago and M. F. López, Nanotechnology, 2010, 21, 405702.
49 J. P. Lewis, P. Jelínek, J. Ortega, A. A. Demkov, D. G. Trabada, B. Haycock, H. Wang, G. Adams, J. K. Tomfohr and E. Abad, et al., Phys. Status Solidi B, 2011, 248, 1989-2007.

50 O. F. Sankey and J. D. Niklewski, Phys. Rev. B: Condens. Matter Mater. Phys., 1989, 40, 3979.

51 Q. Yuan, X. Zhu, K. Lin and Y.-P. Zhao, Phys. Chem. Chem. Phys., 2015, 17, 31887-31893.

52 K. S. Subrahmanyama, P. Kumara, U. Maitraa, A. Govindaraja, K. P. Hembramb, U. V. Waghmareb and C. N. R. Raoa, Proc. Natl. Acad. Sci. U. S. A., 2011, 108, 2674.

53 I. Horcas, R. Fernández, J. M. Gómez-Rodríguez, J. Colchero, J. Gómez-Herrero and A. M. Baró, Rev. Sci. Instrum., 2007, 78, 013705, WSxM solutions website www.wsxmsolutions.com.

54 B. Fultz, Prog. Mater. Sci., 2010, 55, 247-352.

55 D. D. Wagman, W. H. Evans, V. B. Parker, R. H. Schumm and I. Halow, The NBS Tables of Chemical Thermodynamic Properties: Selected Values for Inorganic and $C 1$ and $C 2$ Organic Substances in SI Units, American Chemical Society and the American Institute of Physics for the National Bureau of Standards, 1982.

56 P. C. Snijders, E. J. Moon, C. González, S. Rogge, J. Ortega, F. Flores and H. H. Weitering, Phys. Rev. Lett., 2007, 99, 116102.

57 P. M. Derlet, D. Nguyen-Manh and S. L. Dudarev, Phys. Rev. B: Condens. Matter Mater. Phys., 2007, 76, 054107.

58 Q. Yuan, Y.-P. Zhao, L. Li and T. Wang, J. Phys. Chem. C, 2009, 113, 6107-6113, charge transfer.

59 H. Schweiger, P. Raybaud, G. Kresse and H. Toulhoat, J. Catal., 2002, 207, 76-87.

60 J. P. Paul and E. Payen, J. Phys. Chem. B, 2003, 107, 4057-4064.

61 F. Dumeignil, J.-F. Paul, E. W. Qian, A. Ishihara, E. Payen and T. Kabe, Res. Chem. Intermed., 2003, 2, 589-607. 